\title{
Evolution of compressible and incompressible fluids separated by a closed interface
}

\author{
I. V. DENISOVA \\ Institute of Mechanical Engineering Problems, Russian Academy of Sciences, V.O., Bol'shoy \\ pr.,61, St.Petersburg 199178, Russia
}

[Received 21 March 1999 and in revised form 24 February 2000]

\begin{abstract}
This work solves the problem governing the simultaneous motion of two viscous liquids of different kinds: compressible and incompressible. The boundary between the fluids is considered as an unknown (free) interface where the surface tension is taken into account. Although the fluids occupy the whole space $\mathbb{R}^{3}$, one of them should have a finite volume.

Local (in time) unique solvability of this problem is obtained in the Sobolev-Slobodetskir spaces of functions. Estimates of the solution of a model problem for the Stokes equations are considered in detail, the interface between the fluids being a plane. The Schauder method is used to study a linear problem with a compact boundary. The passage to the nonlinear problem is made by successive approximations.
\end{abstract}

Keywords: free boundary problem, Navier-Stokes equations, two immiscible fluids

\section{Introduction}

This paper considers the unsteady motion of a bubble in an incompressible fluid, or that of a drop in a compressible one. On one hand, the study of a simultaneous motion of two viscous capillary liquids of different kinds (compressible and incompressible) is interesting from the pure mathematical point of view. The present work can be considered as a continuation of [2-5] where the evolution of two viscous capillary fluids of single type, two compressible [4], or two incompressible [2,3,5] have been considered. On the other hand, this problem arises in many important physical phenomena. The evolution of a bubble in an incompressible fluid appears, for example, in the case of an ejection of gas into water, for example, after an explosion in the ocean or after a volcanic eruption at the ocean bed. Another example of the physical interpretation of our problem may be the presence of many small bubbles in a big volume of liquid with large distances between them. Then we can also consider the motion of a single bubble in an infinite liquid medium. A similar situation arises for drops in a volume of gas.

Here, we study the problem governing the motion of two different liquids in full generality. The local unique solvability of this free boundary problem is the main result of the present work. An essential difference in the proof of the existence of a unique solution to the problem is contained in the analysis of a linear model problem with a plane interface between the fluids, so we examine this model problem in detail. Section 2 is devoted to the homogeneous problem and Section 3 deals with the non-homogeneous one. We describe briefly the scheme of the nonlinear problem in Section 4. Its key features are the same as in the case of a single type fluid. The detailed proofs can be found in articles [7-9] where the motion of a finite volume of a single viscous capillary fluid was analysed.

We obtain our results under some restrictions (the relations (1.9)) for the coefficients of the liquid viscosities. These inequalities are imposed for mathematical reasons but are physically reasonable 
in certain cases. They are satisfied, for instance, for the pairs air-water, air-alcohol, air-mercury, etc. but not for air-glycerin, air-lubricating oil. More generally, they are valid for fluids with low viscosity. Incompressible liquids with large viscosity at ordinary temperatures may also satisfy (1.9) when the temperature increases above certain values, lowering the viscosity. This is because for capillary liquids, both viscosities $\mu$ and $v$ decrease quickly with temperature raising whereas for gases, conversely, $\mu$ and $v$ increase with the rise of temperature (see for example [6]). For instance, inequalities (1.9) become true for the pair lubricating oil and air at temperature $\approx 80^{\circ} \mathrm{C}$.

We consider, for definiteness, the case when at the initial moment $t=0$ a compressible fluid is situated in an interior, bounded, domain $\Omega_{0}^{+} \subset \mathbb{R}^{3}$. Let $\mu^{+}>0, \lambda^{+}>0$ be its kinematic viscosities. Let the 'exterior' domain $\Omega_{0}^{-} \equiv \mathbb{R}^{3} \backslash \overline{\Omega_{0}^{+}}$be occupied by an incompressible fluid with the kinematic viscosity $v^{-}>0$ and density $\rho^{-}>0$. We assume that the compressible fluid is barotropic. We note that we could also assume the compressible fluid to be exterior to the incompressible one.

The problem consists in determining, for each $t>0$, the free interface $\Gamma_{t}$ between the liquids evolving in the domains $\Omega_{t}^{-}$and $\Omega_{t}^{+}$. In addition, it is necessary to find the density function $\rho^{+}(x, t)>0$ of the compressible fluid, the pressure function $p^{-}(x, t)$ of the incompressible fluid, as well as the velocity vector field of both liquids $\boldsymbol{v}(x, t)=\left(v_{1}, v_{2}, v_{3}\right)$ satisfying the initial-boundary value problem for the Navier-Stokes system:

$$
\begin{gathered}
\rho^{+}\left(\mathcal{D}_{t} \boldsymbol{v}+(\boldsymbol{v} \cdot \nabla) \boldsymbol{v}\right)-\nabla \mathbb{T}=\rho^{+} \boldsymbol{f}, \\
\mathcal{D}_{t} \rho^{+}+\nabla \cdot\left(\rho^{+} \boldsymbol{v}\right)=0 \quad \text { in } \quad \Omega_{t}^{+}, \quad t>0, \\
\left.\rho^{+}\right|_{t=0}=\rho_{0}^{+},\left.\quad \boldsymbol{v}\right|_{t=0}=\boldsymbol{v}_{0} \quad \text { in } \quad \Omega_{0}^{+}, \\
\mathcal{D}_{t} \boldsymbol{v}-v^{-} \nabla^{2} \boldsymbol{v}+(\boldsymbol{v} \cdot \nabla) \boldsymbol{v}+\frac{1}{\rho^{-}} \nabla p^{-}=\boldsymbol{f}, \quad \nabla \cdot \boldsymbol{v}=0 \quad \text { in } \quad \Omega_{t}^{-}, \quad t>0, \\
\left.\boldsymbol{v}\right|_{t=0}=\boldsymbol{v}_{0} \quad \text { in } \quad \Omega_{0}^{-} ; \quad \boldsymbol{v} \underset{|x| \rightarrow \infty}{\longrightarrow} 0, \quad p^{-} \underset{|x| \rightarrow \infty}{\longrightarrow} 0 ; \\
{\left.[\boldsymbol{v}]\right|_{\Gamma_{t}} \equiv \lim _{\substack{x \rightarrow x_{0} \in \Gamma_{t}, x \in \Omega_{t}^{+}}} \boldsymbol{v}(x)-\lim _{\substack{x \rightarrow x_{0} \in \Gamma_{t}, x \in \Omega_{t}^{-}}} \boldsymbol{v}(x)=0,} \\
{\left.[\mathbb{T} \boldsymbol{n}]\right|_{\Gamma_{t}}=\sigma H \boldsymbol{n} \text { on } \quad \Gamma_{t}, \quad t>0 .}
\end{gathered}
$$

Here $\mathcal{D}_{t}=\partial / \partial t, \quad \nabla=\left(\partial / \partial x_{1}, \partial / \partial x_{2}, \partial / \partial x_{3}\right), \nabla^{2}=\nabla \cdot \nabla$, and the stress tensor is given by

$$
\mathbb{T}= \begin{cases}\left(-p^{+}\left(\rho^{+}\right)+\lambda \nabla \cdot \boldsymbol{v}\right) \mathbb{I}+\mu^{+} \mathbb{S}(\boldsymbol{v}) & \text { in } \Omega_{t}^{+}, \\ -p^{-} \mathbb{I}+\mu^{-} \mathbb{S}(\boldsymbol{v}) & \text { in } \Omega_{t}^{-},\end{cases}
$$

$(\mathbb{S}(\boldsymbol{v}))_{i k}=\partial v_{i} / \partial x_{k}+\partial v_{k} / \partial x_{i}, \quad i, k=1,2,3 ; \quad \mathbb{I}$ is the unit matrix; $\mu^{-}=v^{-} \rho^{-} ; \quad p^{+}\left(\rho^{+}\right)$ is the pressure of the compressible fluid given by a smooth function of its density; $\boldsymbol{f}$ is the given vector field of mass forces; $\boldsymbol{v}_{0}$ is the initial value of the velocity vector field; $\rho_{0}^{+}$is the initial density distribution of the compressible fluid; $\sigma \geqslant 0$ is the surface tension coefficient, $\boldsymbol{n}$ is the outward normal vector to $\Omega_{t}^{+}, H(x, t)$ is twice the mean curvature of $\Gamma_{t}\left(H<0\right.$ at the points where $\Gamma_{t}$ is convex towards $\Omega_{t}^{-}$); $\nabla \mathbb{T}$ means the vector with the components

$$
(\nabla \mathbb{T})_{j}=\frac{\partial T_{i j}}{\partial x_{i}}, \quad T_{i j}=(\mathbb{T})_{i j}, \quad j=1,2,3 .
$$


We use the standard convention on the summation from 1 to 3 with respect to repeated indices. A Cartesian coordinate system $\{x\}$ is introduced in $\mathbb{R}^{3}$.

Since we suppose the liquids to be immiscible, it is natural to impose on $\Gamma_{t}$ a condition excluding the mass transportation through this surface. Mathematically, this condition means that $\Gamma_{t}$ consists of the points $x(\xi, t)$ whose radius vector $\boldsymbol{x}(\xi, t)$ is a solution of the Cauchy problem

$$
\mathcal{D}_{t} \boldsymbol{x}=\boldsymbol{v}(x(\xi, t), t), \quad \boldsymbol{x}(\xi, 0)=\boldsymbol{\xi}, \quad \xi \in \Gamma, \quad t>0,
$$

where $\Gamma \equiv \Gamma_{0}=\partial \Omega_{0}^{+}$is a surface given at the initial moment. Hence, $\Omega_{t}^{ \pm}=\{x=x(\xi, t) \mid \xi \in$ $\left.\Omega_{0}^{ \pm}\right\}$

The condition (1.4) completes the system (1.1)-(1.3).

As usual, we transform the Eulerian coordinates $\{x\}$ into the Lagrangian ones $\{\xi\}$ by the formula

$$
\boldsymbol{x}(\xi, t)=\boldsymbol{\xi}+\int_{0}^{t} \boldsymbol{u}(\xi, \tau) \mathrm{d} \tau \equiv \boldsymbol{X}_{\boldsymbol{u}}(\xi, t)
$$

Here $\boldsymbol{u}(\xi, t)$ is the velocity vector field in the Lagrangian coordinates.

The Jacobian of the transformation (1.5)

$$
\begin{gathered}
\mathcal{J}_{\boldsymbol{u}}(\xi, t)=\operatorname{det}\left\{a_{i j}\right\}_{i, j=1}^{3}, \\
a_{i j}(\xi, t)=\delta_{j}^{i}+\int_{0}^{t} \frac{\partial u_{i}}{\partial \xi_{j}} \mathrm{~d} \tau,
\end{gathered}
$$

being a solution of the Cauchy problem

$$
\begin{gathered}
\mathcal{D}_{t} \mathcal{J}_{\boldsymbol{u}}(\xi, t)=A_{i j} \frac{\partial u_{i}}{\partial \xi_{j}} \equiv \mathcal{J}_{\boldsymbol{u}}(\xi, t)\left(\left.\nabla \cdot \boldsymbol{v}\right|_{x=X_{\boldsymbol{v}}}\right), \\
\mathcal{J}_{\boldsymbol{u}}(\xi, 0)=1
\end{gathered}
$$

may be expressed by the formula

$$
\mathcal{J}_{\boldsymbol{u}}(\xi, t)=\exp \left(\left.\int_{0}^{t} \nabla \cdot \boldsymbol{v}\right|_{x=X_{\boldsymbol{u}}} \mathrm{d} \tau\right) \equiv \exp \left(\int_{0}^{t} \nabla_{\boldsymbol{u}} \cdot \boldsymbol{u} \mathrm{d} \tau\right)
$$

Here $\left\{\delta_{j}^{i}\right\}_{i, j=1}^{3}$ denotes the Kronecker symbol,

$$
\nabla_{\boldsymbol{u}} \equiv\left\{\frac{\partial \xi_{i}}{\partial x_{k}} \frac{\partial}{\partial \xi_{i}}\right\}_{k=1}^{3}=\mathcal{J}_{\boldsymbol{u}}^{-1} \mathbb{A} \nabla
$$

and $\mathbb{A} \equiv\left\{A_{i j}\right\}_{i, j=1}^{3}$ is the cofactor matrix for the Jacobi matrix $\left\{a_{i j}\right\}$ of (1.5). We remark that $\mathcal{J}_{\boldsymbol{u}}(\xi, t) \equiv 1$ in the domain $\Omega_{t}^{-}$.

After the tranformation (1.5) of the system (1.1)-(1.3), the second equation in (1.1) in Lagrangean coordinates takes the form

$$
\mathcal{D}_{t} \widehat{\rho^{+}}+\widehat{\rho^{+}} \nabla_{\boldsymbol{u}} \cdot \boldsymbol{u}=0
$$

from where, in virtue of (1.6), we obtain the following expression for the density $\widehat{\rho^{+}}$

$$
\widehat{\rho^{+}}(\xi, t)=\rho_{0}^{+}(\xi) \exp \left(-\int_{0}^{t} \nabla_{\boldsymbol{u}} \cdot \boldsymbol{u} \mathrm{d} \tau\right)=\rho_{0}^{+}(\xi) \mathcal{J}_{\boldsymbol{u}}^{-1}(\xi, t) .
$$


We substitute (1.7) in the first equation of (1.1) and apply the well-known formula

$$
H \boldsymbol{n}=\Delta(t) \boldsymbol{x}=\Delta(t) \boldsymbol{X}_{\boldsymbol{u}}
$$

where $\Delta(t)$ is the Beltrami-Laplace operator on $\Gamma_{t}$. Moreover, we separate the last vector boundary condition in (1.3) in the tangential and in the normal components. To this end, we project it first onto the tangent plane of $\Gamma_{t}$ and then onto that of $\Gamma$ by means of projectors $\Pi$ and $\Pi_{0}$, respectively.

Next, let $\boldsymbol{n}_{0}$ be the outward normal to $\Gamma$. It is connected with $\boldsymbol{n}$ by the relation

$$
\boldsymbol{n}=\frac{\mathcal{J}_{\boldsymbol{u}}^{-1} \mathbb{A} \boldsymbol{n}_{0}}{\left|\mathcal{J}_{\boldsymbol{u}}^{-1} \mathbb{A} \boldsymbol{n}_{0}\right|}=\frac{\mathbb{A} \boldsymbol{n}_{0}}{\left|\mathbb{A} \boldsymbol{n}_{0}\right|}
$$

For $\boldsymbol{n} \cdot \boldsymbol{n}_{0}>0$, problem (1.1)-(1.4), as a result of the above transformation, is changed into an equivalent system:

$$
\begin{aligned}
& \mathcal{D}_{t} \boldsymbol{u}-\frac{1}{\rho_{0}^{+}(\xi)} \mathbb{A} \nabla \mathbb{T}_{\boldsymbol{u}}^{\prime}(\boldsymbol{u})=\boldsymbol{f}\left(X_{\boldsymbol{u}}, t\right)-\frac{1}{\rho_{0}^{+}(\xi)} \mathbb{A} \nabla p^{+}\left(\rho_{0}^{+} \mathcal{J}_{\boldsymbol{u}}^{-1}\right) \\
& \text { in } Q_{T}^{+} \equiv \Omega_{0}^{+} \times(0, T) \\
& \mathcal{D}_{t} \boldsymbol{u}-v^{-} \nabla_{\boldsymbol{u}}^{2} \boldsymbol{u}+\frac{1}{\rho^{-}} \nabla_{\boldsymbol{u}} q=\boldsymbol{f}\left(X_{\boldsymbol{u}}, t\right), \\
& \nabla_{\boldsymbol{u}} \cdot \boldsymbol{u}=0 \quad \text { in } \quad Q_{T}^{-} \equiv \Omega_{0}^{-} \times(0, T), \\
& \left.\boldsymbol{u}\right|_{t=0}=\boldsymbol{v}_{0} \quad \text { in } \quad \Omega_{0}^{-} \cup \Omega_{0}^{+}, \quad \boldsymbol{u} \underset{|\xi| \rightarrow \infty}{\longrightarrow} 0, \quad q \underset{|\xi| \rightarrow \infty}{\longrightarrow} 0, \\
& {\left.[\boldsymbol{u}]\right|_{G_{T}}=0,\left.\quad\left[\mu^{ \pm} \Pi_{0} \Pi \mathbb{S}_{\boldsymbol{u}}(\boldsymbol{u}) \boldsymbol{n}\right]\right|_{G_{T}}=0 \quad\left(G_{T} \equiv \Gamma \times(0, T)\right),} \\
& {\left.\left[\boldsymbol{n}_{0} \cdot \mathbb{T}_{\boldsymbol{u}}^{\prime}(\boldsymbol{u}, q) \boldsymbol{n}\right]\right|_{G_{T}}-\left.\sigma \boldsymbol{n}_{0} \cdot \Delta(t) \boldsymbol{X}_{\boldsymbol{u}}\right|_{G_{T}}=\left.\left(\boldsymbol{n}_{0} \cdot \boldsymbol{n}\right) p^{+}\left(\rho_{0}^{+} \mathcal{J}_{\boldsymbol{u}}^{-1}\right)\right|_{G_{T}} .}
\end{aligned}
$$

In (1.8) $q(\xi, t)$ was the pressure function in the Lagrangean coordinates, and we used the following notation:

$$
\begin{aligned}
& \left(\mathbb{T}_{\boldsymbol{u}}^{\prime}(\boldsymbol{w}, q)\right)_{i, j}= \begin{cases}\left(\lambda^{+} \nabla_{\boldsymbol{u}} \cdot \boldsymbol{w}\right) \delta_{j}^{i}+\mu^{+}\left(\mathbb{S}_{\boldsymbol{u}}(\boldsymbol{w})\right)_{i j} & \text { in } Q_{T}^{+}, \\
-\delta_{j}^{i} q+\mu^{-}\left(\mathbb{S}_{\boldsymbol{u}}(\boldsymbol{w})\right)_{i j} & \text { in } Q_{T}^{-},\end{cases} \\
& \left(\mathbb{S}_{\boldsymbol{u}}(\boldsymbol{w})\right)_{i j}=\mathcal{J}_{\boldsymbol{u}}^{-1}\left(A_{i k} \frac{\partial w_{j}}{\partial \xi_{k}}+A_{j k} \frac{\partial w_{i}}{\partial \xi_{k}}\right) ; \\
& \Pi_{0} \boldsymbol{\omega}=\boldsymbol{\omega}-\left(\boldsymbol{n}_{0} \cdot \boldsymbol{\omega}\right) \boldsymbol{n}_{0}, \quad \Pi \boldsymbol{\omega}=\boldsymbol{\omega}-(\boldsymbol{n} \cdot \boldsymbol{\omega}) \boldsymbol{n} .
\end{aligned}
$$

The main result of this paper is a theorem on the unique solvability of problem (1.8) in the Sobolev-Slobodetskii spaces. We now recall the definition of these spaces.

Let $\Omega$ be a domain in $\mathbb{R}^{n}, n \in \mathbb{N}$, and let $\boldsymbol{\alpha}=\left(\alpha_{1}, \ldots, \alpha_{n}\right)$ be the multi-index of order $|\boldsymbol{\alpha}|=\alpha_{1}+\cdots+\alpha_{n}$ with integer non-negative components $\alpha_{i}, i=1, \ldots, n$. We denote the generalized derivative of a function $u$ by $\mathcal{D}_{x}^{\alpha} u=\frac{\partial^{|\alpha|} u}{\partial x_{1}^{\alpha_{1}} \ldots \partial x_{n}^{\alpha_{n}}}$.

We determine the Sobolev-Slobodetskiǐ space $W_{2}^{m}(\Omega)$ for $m>0$ as the space of functions $u$ with finite norm

$$
\|u\|_{W_{2}^{m}(\Omega)}=\left(\sum_{|\alpha|<m}\left\|\mathcal{D}_{x}^{\alpha} u\right\|_{\Omega}^{2}+\|u\|_{\dot{W}_{2}^{m}(\Omega)}^{2}\right)^{1 / 2}
$$


where $\|\cdot\|_{\Omega}$ is the norm of $L_{2}(\Omega)$ and

$$
\|u\|_{\dot{W}_{2}^{m}(\Omega)}^{2}= \begin{cases}\sum_{|\alpha|=m}\left\|\mathcal{D}_{x}^{\alpha} u\right\|_{\Omega}^{2} & \text { for } m \in \mathbb{N}, \\ \sum_{|\alpha|=[m]} \int_{\Omega} \int_{\Omega} \frac{\left|\mathcal{D}_{x}^{\alpha} u(x)-\mathcal{D}_{y}^{\alpha} u(y)\right|^{2}}{|x-y|^{n+2(m-[m])}} \mathrm{d} x \mathrm{~d} y & \text { for } m \notin \mathbb{N},\end{cases}
$$

$[m]$ being the integer part of $m$.

The anisotropic space $W_{2}^{m, m / 2}\left(Q_{T}\right)$ consists of functions defined in the cylinder $Q_{T}=\Omega \times$ $(0, T), 0<T \leqslant \infty$, and having finite norm

$$
\|u\|_{W_{2}^{m, m / 2}\left(Q_{T}\right)}=\left(\int_{0}^{T}\|u\|_{W_{2}^{m}(\Omega)}^{2} \mathrm{~d} t+\int_{\Omega}\|u\|_{W_{2}^{m / 2}(0, T)}^{2} \mathrm{~d} x\right)^{1 / 2} .
$$

To formulate intermediate results, we need the Sobolev-Slobodetskiř spaces with exponential weight introduced by M. Agranovich and M. Vishik in [1] and also considered by V. Solonnikov in [7].

Let $\gamma \geqslant 0$. The weighted space $H_{\gamma}^{m, m / 2}\left(Q_{T}\right)$ is the space of functions admitting zero continuation to the domain $t<0$ without loss of regularity. This space is equipped with the norm

$$
\|u\|_{H_{\gamma}^{m, m / 2}\left(Q_{T}\right)}=\left(\|u\|_{H_{\gamma}^{m, 0}\left(Q_{T}\right)}^{2}+\|u\|_{H_{\gamma}^{0, m / 2}\left(Q_{T}\right)}^{2}\right)^{1 / 2} .
$$

Here

$$
\begin{aligned}
& \|u\|_{H_{\gamma}^{m, 0}\left(Q_{T}\right)}^{2}=\int_{0}^{T} \mathrm{e}^{-2 \gamma t}\left(\|u\|_{\dot{W}_{2}^{m}(\Omega)}^{2}+\gamma^{m}\|u\|_{\Omega}^{2}\right) \mathrm{d} t, \\
& \|u\|_{H_{\gamma}^{0, m / 2}\left(Q_{T}\right)}^{2}=\int_{0}^{T} \mathrm{e}^{-2 \gamma t}\left\|\frac{\partial^{m / 2} u}{\partial t^{m / 2}}\right\|_{\Omega}^{2} \mathrm{~d} t \quad \text { for } m / 2 \in \mathbb{N}
\end{aligned}
$$

and

$$
\|u\|_{H_{\gamma}^{0, m / 2}\left(Q_{T}\right)}^{2}=\int_{0}^{T} \mathrm{e}^{-2 \gamma t} \int_{0}^{\infty}\left\|\frac{\partial^{k} u_{0}(\cdot, t)}{\partial t^{k}}-\frac{\partial^{k} u_{0}(\cdot, t-\tau)}{\partial t^{k}}\right\|_{\Omega}^{2} \frac{\mathrm{d} \tau}{\tau^{1+m-2 k}} \mathrm{~d} t
$$

for $k \equiv[m / 2]<m / 2$ where $u_{0}$ is the extension of $u$ by zero in the domain $t<0$. In addition, in the case $m>1$,

$$
\left.\frac{\partial^{i} u}{\partial t^{i}}\right|_{t=0}=0, \quad i=0, \ldots,\left[\frac{m-1}{2}\right] .
$$

On the cylinder boundary $G_{T}=\partial \Omega \times(0, T)$ of the domain $Q_{T}$, we define the space $H_{\gamma}^{m+1 / 2,1 / 2, m / 2}\left(G_{T}\right)$ with the norm whose square is determined by the formula

$$
\begin{array}{r}
\|u\|_{H_{\gamma}^{m+1 / 2,1 / 2, m / 2}\left(G_{T}\right)}^{2}=\int_{0}^{T} \mathrm{e}^{-2 \gamma t}\left(\|u\|_{W_{2}^{m+1 / 2}(\partial \Omega)}^{2}+\gamma^{m}\|u\|_{W_{2}^{1 / 2}(\partial \Omega)}^{2}\right. \\
\left.\quad+\int_{0}^{\infty}\left\|\frac{\partial^{k} u_{0}(\cdot, t)}{\partial t^{k}}-\frac{\partial^{k} u_{0}(\cdot, t-\tau)}{\partial t^{k}}\right\|_{W_{2}^{1 / 2}(\partial \Omega)}^{2} \frac{\mathrm{d} \tau}{\tau^{1+m-2 k}}\right) \mathrm{d} t
\end{array}
$$


$k \equiv[m / 2]<m / 2$; if $k=m / 2$ the last term under the first integral sign should be changed into $\left\|\frac{\partial^{m / 2} u}{\partial t^{m / 2}}\right\|_{W_{2}^{1 / 2}(\partial \Omega)}^{2}$. (Here $u_{0}$ is again the extension of $u$ by zero and $\left.\frac{\partial^{i} u}{\partial t^{i}}\right|_{t=0}=0, \quad i=$ $\left.0, \ldots,\left[\frac{m-1}{2}\right].\right)$

All these norms can be introduced on any smooth manifold by means of local maps and partitions of unity.

As usual, for a function defined in two domains $Q_{T}^{-}$and $Q_{T}^{+}$, we set

$$
\|u\|_{\bigcup_{i=-,+} H_{\gamma}^{m, m / 2}\left(Q_{T}^{i}\right)}=\|u\|_{H_{\gamma}^{m, m / 2}\left(Q_{T}^{-}\right)}+\|u\|_{H_{\gamma}^{m, m / 2}\left(Q_{T}^{+}\right)} .
$$

We say that a vector field belongs to a certain space if each of its components belongs to this space and we define its norm as the sum of the norms of its components. The numeration of constants is individual for each section.

Now we define three norms necessary for formulating the main result of this paper. The first of them is

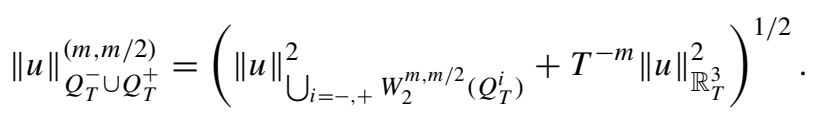

It is equivalent to $\|u\|_{\bigcup_{i} H_{\gamma}^{m, m / 2}\left(Q_{T}^{i}\right)}^{2}$ if $m<1$ and to $\|u\|_{\bigcup_{i} W_{2}^{m, m / 2}\left(Q_{T}^{i}\right)}^{2}$ for $\forall T<\infty$. The square of the second norm is determined by the formula

$$
\begin{array}{r}
\left(\|u\|_{Q_{T}^{-} \cup Q_{T}^{+}}^{(2+l, 1+l / 2)}\right)^{2}=\|u\|_{\bigcup_{i=-,+}}^{2} W_{2}^{2+l, 1+l / 2}\left(Q_{T}^{i}\right) \\
\left.+\sum_{|\alpha|=2}\left\|\mathcal{D}_{x}^{\alpha} u\right\|_{Q_{T}^{-} \cup Q_{T}^{+}}^{2}\right\}+\sup _{t \leqslant T}\|u(\cdot, t)\|_{\bigcup_{i} W_{2}^{1+l}\left(\Omega_{0}^{i}\right)}^{2}
\end{array}
$$

For $\beta \in(0,1)$ we will consider the following Hölder norm of $u \in \mathbb{R}_{T}^{3} \equiv \mathbb{R}^{3} \times(0, T)$ :

$$
\||u|\|_{\mathbb{R}_{T}^{3}}=\sup _{\mathbb{R}_{T}^{3}}|u|+\max _{k} \sup _{(x, t) \in \mathbb{R}_{T}^{3}}\left|\mathcal{D}_{x_{k}} u(x, t)\right|+\sup _{(x, t), \tau \leqslant T} \frac{|u(x, t)-u(x, \tau)|}{|\tau-t|^{\beta}} .
$$

Let $B_{d}$ be the ball $\{x:|x|<d\}$. We choose a coordinate system $\{x\}$ so that $\Omega_{0}^{+}$is contained in the ball $B_{d}, d<\infty$, and we set $B_{d T}^{-} \equiv\left(B_{d} \backslash \overline{\Omega_{0}^{+}}\right) \times(0, T)$.

THEOREM 1.1 Assume that for some $l \in(1 / 2,1)$ we have $\Gamma \in W_{2}^{5 / 2+l}, \rho_{0}^{+} \in W_{2}^{1+l}\left(\Omega_{0}^{+}\right), 0<$ $R_{0} \leqslant \rho_{0}^{+}(\xi) \leqslant R_{\infty}<\infty, \xi \in \Omega_{0}^{+}, p^{+} \in C^{3}\left(\mathbb{R}_{+}\right), \boldsymbol{f} \in \boldsymbol{W}_{2}^{l, l / 2}\left(\mathbb{R}_{T}^{3}\right), 0<T<\infty, \boldsymbol{f}(\cdot, t)$ $\in \boldsymbol{C}^{2}\left(\mathbb{R}^{3}\right)$ for $\forall t \in[0, T], \boldsymbol{f}(\xi, \cdot), \nabla \boldsymbol{f}(\xi, \cdot) \in \boldsymbol{C}^{\beta}(0, T)$ for $\forall \xi \in \mathbb{R}^{3}$ with some $\beta \in(1 / 2,1)$. In addition, let the initial velocity vector $v_{0} \in \bigcup_{i=-,+} W_{2}^{1+l}\left(\Omega_{0}^{i}\right)$ satisfy the compatibility conditions

$$
\begin{gathered}
\nabla \cdot \boldsymbol{v}_{0}=0 \quad \text { in } \Omega_{0}^{-}, \\
{\left.\left[\boldsymbol{v}_{0}\right]\right|_{\Gamma}=0,\left.\quad\left[\Pi_{0} \mathbb{S}\left(\boldsymbol{v}_{0}\right) \boldsymbol{n}_{0}\right]\right|_{\Gamma}=0,}
\end{gathered}
$$

and let the viscosities of the liquids satisfy the inequalities

$$
\mu^{-}>\mu^{+}, \quad v^{-}<\mu^{+} / R_{\infty} .
$$


Under these hypotheses, there exists a constant $T_{0} \in(0, T]$ such that problem (1.8) is uniquely solvable on the interval $\left(0, T_{0}\right)$, and its solution $(\boldsymbol{u}, q)$ has the properties: $\boldsymbol{u} \in$ $\bigcup_{i=-,+} \boldsymbol{W}_{2}^{2+l, 1+l / 2}\left(Q_{T_{0}}^{i}\right), q \in W_{2, l o c}^{l, l / 2}\left(Q_{T_{0}}^{-}\right), \nabla q \in W_{2}^{l, l / 2}\left(Q_{T_{0}}^{-}\right),\left.q\right|_{G_{T_{0}}} \in W_{2}^{l+1 / 2, l / 2+1 / 4}\left(G_{T_{0}}\right)$ and

$$
\begin{aligned}
& \|\boldsymbol{u}\|_{Q_{T_{0}}^{-} \cup Q_{T_{0}}^{+}}^{(2+l, 1+l / 2)}+\|\nabla q\|_{Q_{T_{0}}^{-}}^{(l, l / 2)}+\|q\|_{B_{d T_{0}}^{-}}^{(l, l / 2)}+\|q\|_{W_{2}^{l+1 / 2, l / 2+1 / 4}\left(G_{T_{0}}\right)} \\
& \leqslant c_{1}\left(c_{2}+c_{3} T_{0}^{\frac{1-l}{2}}\left\|\boldsymbol{v}_{0}\right\|_{\cup_{i} \boldsymbol{W}_{2}^{1+l}\left(\Omega_{0}^{i}\right)}\right)\left\{\||\boldsymbol{f}|\|_{\mathbb{R}_{T_{0}}^{3}}+\left\|\boldsymbol{v}_{0}\right\|_{\cup_{i} \boldsymbol{W}_{2}^{1+l}\left(\Omega_{0}^{i}\right)}\right. \\
& \left.\quad+\sigma\left\|H_{0}\right\|_{W_{2}^{l+1 / 2}(\Gamma)}+\left\|\frac{1}{\rho_{0}^{+}} \nabla p^{+}\left(\rho_{0}^{+}\right)\right\|_{W_{2}^{l}\left(\Omega_{0}^{+}\right)}+\left\|p^{+}\left(\rho_{0}^{+}\right)\right\|_{W_{2}^{1+l}\left(\Omega_{0}^{+}\right)}\right\} .
\end{aligned}
$$

The value of $T_{0}$ depends on the norms of $\boldsymbol{f}, \boldsymbol{v}_{0}, \rho_{0}, p^{+}$and on the curvature magnitude of $\Gamma$.

This theorem is proved by successive approximations in the same way as the analogous theorems for the case of a single incompressible fluid [8] or for the case of a single compressible one [9]. We will consider the main steps of the proof in Section 4. The role of the successive approximations will be played by the solutions of the following linearized problems:

$$
\begin{gathered}
\mathcal{D}_{t} \boldsymbol{w}-\frac{1}{\rho_{0}^{+}(\xi)} \mathbb{A} \nabla \mathbb{T}_{\boldsymbol{u}}^{\prime}(\boldsymbol{w})=\boldsymbol{f} \quad \text { in } \quad Q_{T}^{+}, \\
\mathcal{D}_{t} \boldsymbol{w}-v^{-} \nabla_{\boldsymbol{u}}^{2} \boldsymbol{w}+\frac{1}{\rho_{0}^{-}} \nabla_{\boldsymbol{u}} s=\boldsymbol{f}, \quad \nabla_{\boldsymbol{u}} \cdot \boldsymbol{w}=r \quad \text { in } \quad Q_{T}^{-}, \\
\left.\boldsymbol{w}\right|_{t=0}=\boldsymbol{w}_{0} \quad \text { in } \quad \Omega_{0}^{-} \cup \Omega_{0}^{+}, \quad \boldsymbol{w} \underset{|\xi| \rightarrow \infty}{\longrightarrow} 0, \quad s \underset{|\xi| \rightarrow \infty}{\longrightarrow} 0, \\
{\left.[\boldsymbol{w}]\right|_{G_{T}}=0,\left.\quad\left[\mu^{ \pm} \Pi_{0} \Pi \mathbb{S}_{\boldsymbol{u}}(\boldsymbol{w}) \boldsymbol{n}\right]\right|_{G_{T}}=\Pi_{0} a,} \\
{\left.\left[\boldsymbol{n}_{0} \cdot \mathbb{T}_{\boldsymbol{u}}^{\prime}(\boldsymbol{w}, s) \boldsymbol{n}\right]\right|_{\Gamma}-\left.\sigma \boldsymbol{n}_{0} \cdot \Delta(t) \int_{0}^{t} \boldsymbol{w}\right|_{\Gamma} \mathrm{d} \tau=b+\sigma \int_{0}^{t} B \mathrm{~d} \tau, \quad t \in(0, T) .}
\end{gathered}
$$

THEOREM 1.2 Let $\Gamma \in W_{2}^{3 / 2+l}, \rho_{0}^{+} \in W_{2}^{1+l}\left(\Omega_{0}^{+}\right)$, for some $l \in(1 / 2,1)$ and let $0<R_{0} \leqslant$ $\rho_{0}^{+}(\xi) \leqslant R_{\infty}<\infty, \xi \in \Omega_{0}^{+}$. In addition, assume that the vector field $\boldsymbol{u}$ is continuous across the boundary $\Gamma$ and that for some $T<\infty$ it satisfies the inequality

$$
T^{1 / 2}\|\boldsymbol{u}\|_{Q_{T}^{-} \cup Q_{T}^{+}}^{(2+l, 1+l / 2)} \leqslant \delta
$$

with a small number $\delta$. We suppose also that inequalities (1.9) hold for the viscosities $\mu^{ \pm}, v^{-}$.

Then for any $\boldsymbol{f} \in \bigcup_{i=-,+} \boldsymbol{W}_{2}^{l, l / 2}\left(Q_{T}^{i}\right), r \in W_{2}^{1+l, 1 / 2+l / 2}\left(Q_{T}^{-}\right), r=\nabla \cdot \boldsymbol{R}, \boldsymbol{R} \in \boldsymbol{W}_{2}^{0,1+l / 2}\left(Q_{T}^{-}\right)$, $\boldsymbol{w}_{0} \in \bigcup_{i=-,+} \boldsymbol{W}_{2}^{1+l}\left(\Omega_{0}^{i}\right), \boldsymbol{a} \in \boldsymbol{W}_{2}^{l+1 / 2, l / 2+1 / 4}\left(G_{T}\right), \quad b \in W_{2}^{l+1 / 2, l / 2+1 / 4}\left(G_{T}\right)$, and $B \in$ $W_{2}^{l-1 / 2, l / 2-1 / 4}\left(G_{T}\right)$ for which the compatibility conditions

$$
\left.\left[\boldsymbol{w}_{0}\right]\right|_{\Gamma}=0,\left.\quad\left[\mu^{ \pm} \Pi_{0} \mathbb{S}\left(\boldsymbol{w}_{0}\right) \boldsymbol{n}_{0}\right]\right|_{\Gamma}=\left.\Pi_{0} \boldsymbol{a}\right|_{t=0},
$$




$$
\nabla \cdot \boldsymbol{w}_{0}=\left.r\right|_{t=0} \quad \text { on } \quad \Omega_{0}^{-}
$$

hold, there exists a unique solution $(\boldsymbol{w}, s)$ of problem (1.10) such that $\boldsymbol{w} \in$ $\bigcup_{i=-,+} \boldsymbol{W}_{2}^{2+l, 1+l / 2}\left(Q_{T}^{i}\right), s \in W_{2, l o c}^{l, l / 2}\left(Q_{T}^{-}\right), \nabla s \in \boldsymbol{W}_{2}^{l, l / 2}\left(Q_{T}^{-}\right),\left.s\right|_{G_{T}} \in W_{2}^{l+1 / 2, l / 2+1 / 4}\left(G_{T}\right)$, and

$$
\begin{gathered}
\|\boldsymbol{w}\|_{Q_{T}^{-} \cup Q_{T}^{+}}^{(2+l, 1+l / 2)}+\|\nabla s\|_{Q_{T}^{-}}^{(l, l / 2)}+\|s\|_{B_{d T}^{-}}^{(l, l / 2)}+\|s\|_{W_{2}^{l+1 / 2, l / 2+1 / 4}\left(G_{T}\right)} \\
\leqslant c_{1}(T)\left\{\|\boldsymbol{f}\|_{Q_{T}^{-} \cup Q_{T}^{+}}^{(l, l / 2)}+\left\|\boldsymbol{w}_{0}\right\|_{\cup_{i} W_{2}^{1+l}\left(\Omega_{0}^{i}\right)}+\|r\|_{W_{2}^{1+l, 0}\left(Q_{T}^{-}\right)}\right. \\
+\|\boldsymbol{R}\|_{W_{2}^{0,1+l / 2}\left(Q_{T}^{-}\right)}+T^{-l / 2}\left\|\mathcal{D}_{t} \boldsymbol{R}\right\|_{Q_{T}^{-}}+\|\boldsymbol{a}\|_{W_{2}^{l+1 / 2, l / 2+1 / 4}\left(G_{T}\right)} \\
+\|b\|_{W_{2}^{l+1 / 2, l / 2+1 / 4}\left(G_{T}\right)}+T^{-l / 2}\|b\|_{W_{2}^{1 / 2,0}\left(G_{T}\right)} \\
\left.+\sigma\|B\|_{W_{2}^{l-1 / 2, l / 2-1 / 4}\left(G_{T}\right)}\right\}
\end{gathered}
$$

$c_{1}(T)$ being a non-decreasing function of $T$.

The existence of a unique smooth solution of problem (1.10) is based on the analysis of the model problem when $\boldsymbol{u} \equiv 0$ and when the interface $\Gamma$ is a plane.

\section{The homogeneous model problem}

In this section, we consider the problem

$$
\begin{gathered}
\mathcal{D}_{t} \boldsymbol{v}-v^{+} \nabla^{2} \boldsymbol{v}+\frac{1}{\rho_{0}^{+}} \nabla p=0, \quad \nabla \cdot \boldsymbol{v}=0, \quad \text { in } D_{\infty}^{+}=\mathbb{R}_{+}^{3} \times(0, \infty), \\
\mathcal{D}_{t} \boldsymbol{v}-v^{-} \nabla^{2} \boldsymbol{v}-\left(v^{-}+\kappa^{-}\right) \nabla(\nabla \cdot \boldsymbol{v})=0 \quad \text { in } \quad D_{\infty}^{-}=\mathbb{R}_{-}^{3} \times(0, \infty) \\
\left.\boldsymbol{v}\right|_{t=0}=0 \quad \text { on } \quad \mathbb{R}_{-}^{3} \cup \mathbb{R}_{+}^{3}, \quad \boldsymbol{v} \underset{|x| \rightarrow \infty}{\longrightarrow} 0, \quad p \underset{|x| \rightarrow \infty}{\longrightarrow} 0 \\
{\left.[\boldsymbol{v}]\right|_{x_{3}=0}=0, \quad-\left.\left[\mu^{ \pm}\left(\frac{\partial v_{\alpha}}{\partial x_{3}}+\frac{\partial v_{3}}{\partial x_{\alpha}}\right)\right]\right|_{x_{3}=0}=b_{\alpha}\left(x^{\prime}, t\right), \quad \alpha=1,2} \\
-\left.\left(p+\lambda^{-} \nabla \cdot \boldsymbol{v}\right)\right|_{x_{3}=0}+\left.\left[2 \mu^{ \pm} \frac{\partial v_{3}}{\partial x_{3}}\right]\right|_{x_{3}=0}+\left.\sigma \Delta^{\prime} \int_{0}^{t} v_{3}\right|_{x_{3}=0} \mathrm{~d} \tau \\
=b_{3}+\sigma \int_{0}^{t} B \mathrm{~d} \tau \equiv b_{3}^{\prime} \quad \text { on } \mathbb{R}_{\infty}^{2}
\end{gathered}
$$

Here we have used the notation $\mathbb{R}_{ \pm}^{3}=\left\{ \pm x_{3}>0\right\}, \mathbb{R}_{\infty}^{2}=\mathbb{R}^{2} \times(0, \infty), \kappa^{-}=\lambda^{-} / \rho_{0}^{-}, \rho_{0}^{-}=$ constant $>0, v^{-}=\mu^{-} / \rho_{0}^{-}, \Delta^{\prime}=\partial^{2} / \partial x_{1}^{2}+\partial^{2} / \partial x_{2}^{2}, x^{\prime}=\left(x_{1}, x_{2}\right)$.

We take the Fourier transform on the tangent space variables $\left(x_{1}, x_{2}\right)=x^{\prime}$ and the Laplace transform with respect to $t$ given by the formula

$$
\tilde{f}\left(\xi, x_{3}, s\right)=\int_{0}^{\infty} e^{-s t} \int_{\mathbb{R}^{2}} f(x, t) \mathrm{e}^{-\mathrm{i} x^{\prime} \cdot \xi} \mathrm{d} x^{\prime} \mathrm{d} t, \operatorname{Re} s \geqslant 0, \quad \xi=\left(\xi_{1}, \xi_{2}\right) .
$$


For a function $f \in H_{\gamma}^{l, l / 2}\left(D_{\infty}^{ \pm}\right)$, transfomation (2.2) defines an analytic function $\tilde{f}$ in the half-plane $\{\operatorname{Re} s>\gamma\}$.

The problem (2.1) is then transformed into the system of ordinary differential equations

$$
\begin{aligned}
& \frac{\mathrm{d}^{2} \widetilde{v}_{\alpha}}{\mathrm{d} x_{3}^{2}}-\left(\frac{s}{v^{+}}+\xi^{2}\right) \widetilde{v}_{\alpha}-\frac{\mathrm{i} \xi_{\alpha}}{\mu^{+}} \widetilde{p}=0, \quad \alpha=1,2, \\
& \frac{\mathrm{d}^{2} \widetilde{v}_{3}}{\mathrm{~d} x_{3}^{2}}-\left(\frac{s}{v^{+}}+\xi^{2}\right) \widetilde{v}_{3}-\frac{1}{\mu^{+}} \frac{\widetilde{p}}{\mathrm{~d} x_{3}}=0, \\
& \frac{\mathrm{d} \widetilde{v}_{3}}{\mathrm{~d} x_{3}}+\mathrm{i} \xi_{\alpha} \widetilde{v}_{\alpha}=0, \\
& \frac{\mathrm{d}^{2} \widetilde{v}_{\alpha}}{\mathrm{d} x_{3}^{2}}-\left(\frac{s}{v^{-}}+\xi^{2}\right) \widetilde{v}_{\alpha}+\left(1+\beta^{-}\right) \mathrm{i} \xi_{\alpha}\left(\mathrm{i} \xi_{1} \widetilde{v}_{1}+\mathrm{i} \xi_{2} \widetilde{v}_{2}+\frac{\mathrm{d} \widetilde{v}_{3}}{\mathrm{~d} x_{3}}\right)=0, \quad \alpha=1,2, \\
& \left(2+\beta^{-}\right) \frac{\mathrm{d}^{2} \widetilde{v}_{3}}{\mathrm{~d} x_{3}^{2}}-\left(\frac{s}{v^{-}}+\xi^{2}\right) \widetilde{v}_{3}+\left(1+\beta^{-}\right) \frac{\mathrm{d}}{\mathrm{d} x_{3}}\left(\mathrm{i} \xi_{1} \widetilde{v}_{1}+\mathrm{i} \xi_{2} \widetilde{v}_{2}\right)=0, \quad x_{3}<0,
\end{aligned}
$$

with the boundary conditions

$$
\begin{aligned}
& \widetilde{\boldsymbol{v}} \underset{\left|x_{3}\right| \rightarrow \infty}{\longrightarrow} 0, \quad \widetilde{p} \underset{\left|x_{3}\right| \rightarrow \infty}{\longrightarrow} 0,\left.\quad[\widetilde{\boldsymbol{v}}]\right|_{x_{3}=0}=0, \\
& -\left.\left[\mu^{ \pm}\left(\frac{\mathrm{d} \widetilde{v}_{\alpha}}{\mathrm{d} x_{3}}+\mathrm{i} \xi_{\alpha} \widetilde{v}_{3}\right)\right]\right|_{x_{3}=0}=\widetilde{b}_{\alpha}, \quad \alpha=1,2, \\
& s\left(-\widetilde{p}^{+}+\left.\left[2 \mu^{ \pm} \frac{\mathrm{d} \widetilde{v}_{3}}{\mathrm{~d} x_{3}}\right]\right|_{x_{3}=0}-\lambda^{-}\left(\sum_{\beta=1}^{2} \mathrm{i} \xi_{\beta} \widetilde{v}_{\beta}^{-}+\frac{\mathrm{d} \widetilde{v}_{3}^{-}}{\mathrm{d} x_{3}}\right)\right)-\sigma \xi^{2} \widetilde{v}_{3}^{+}=s \widetilde{b}_{3}^{\prime}
\end{aligned}
$$

where $\xi^{2}=\xi_{1}^{2}+\xi_{2}^{2}, \beta^{-} \equiv \kappa^{-} / v^{-} \equiv \lambda^{-} / \mu^{-}, \widetilde{\boldsymbol{v}}^{ \pm}=\lim _{x_{3} \rightarrow 0 \pm} \widetilde{\boldsymbol{v}}, \quad \widetilde{p}^{+}=\lim _{x_{3} \rightarrow 0+} \widetilde{p}$.

As in the case of two one-type liquids (see $[2,4]$ ), we write the general solution of $(2.3)$ in the two half-spaces:

$$
\begin{aligned}
& \tilde{\boldsymbol{v}}=C_{1}^{+}\left(\begin{array}{c}
r^{+} \\
0 \\
\mathrm{i} \xi_{1}
\end{array}\right) \mathrm{e}^{-r^{+} x_{3}}+C_{2}^{+}\left(\begin{array}{c}
0 \\
r^{+} \\
\mathrm{i} \xi_{2}
\end{array}\right) \mathrm{e}^{-r^{+} x_{3}}+C_{3}^{+}\left(\begin{array}{c}
\mathrm{i} \xi_{1} \\
\mathrm{i} \xi_{2} \\
-|\xi|
\end{array}\right) \mathrm{e}^{-|\xi| x_{3}}, \\
& \tilde{p}=-C_{3}^{+} \rho_{0}^{+} s \mathrm{e}^{-|\xi| x_{3}} \\
& \text { for } x_{3}>0, \\
& \tilde{\boldsymbol{v}}=C_{1}^{-}\left(\begin{array}{c}
-r^{-} \\
0 \\
\mathrm{i} \xi_{1}
\end{array}\right) \mathrm{e}^{r^{-} x_{3}}+C_{2}^{-}\left(\begin{array}{c}
0 \\
-r^{-} \\
\mathrm{i} \xi_{2}
\end{array}\right) \mathrm{e}^{r^{-} x_{3}}+C_{3}^{-}\left(\begin{array}{c}
\mathrm{i} \xi_{1} \\
\mathrm{i} \xi_{2} \\
r_{1}^{-}
\end{array}\right) \mathrm{e}^{r_{1}^{-} x_{3}} \\
& \text { for } x_{3}<0 .
\end{aligned}
$$

Here $C_{i}^{ \pm}, i=1,2,3$, are arbitrary constants, $r^{ \pm}=\sqrt{\frac{s}{v^{ \pm}}+\xi^{2}}, r_{1}^{-}=\sqrt{\frac{s}{\left(2+\beta^{-}\right) v^{-}}+\xi^{2}},|\xi|=$ $\sqrt{\xi_{1}^{2}+\xi_{2}^{2}}$ and we assume $|\arg \sqrt{z}|<\pi / 2$ for $\forall z \in \mathbb{C}$.

We substitute (2.5) in the boundary conditions (2.4) and solve the system obtained. Then we find 
a solution of problem (2.3), (2.4) which we write in the form convenient for the following estimates:

$$
\begin{aligned}
& \tilde{\boldsymbol{v}}=\boldsymbol{W} e_{0}^{ \pm}+\boldsymbol{V}^{ \pm} e_{1}^{ \pm}, \quad \pm x_{3}>0, \\
& \tilde{p}=-C_{3}^{+} \rho_{0}^{+} \mathrm{s}^{-|\xi| x_{3}}=-\mu^{+} C_{3}^{+}\left(r^{+}-|\xi|\right)\left(r^{+}+|\xi|\right) \mathrm{e}^{-|\xi| x_{3}}, \quad x_{3}>0,
\end{aligned}
$$

where

$$
\begin{aligned}
& e_{0}^{ \pm}=\mathrm{e}^{\mp r^{ \pm} x_{3}}, \quad e_{1}^{+}=\frac{\mathrm{e}^{-r^{+} x_{3}}-\mathrm{e}^{-|\xi| x_{3}}}{r^{+}-|\xi|}, \quad e_{1}^{-}=\frac{\mathrm{e}^{r^{-} x_{3}}-\mathrm{e}_{1}^{r_{1}^{-} x_{3}}}{r^{-}-r_{1}^{-}}, \\
& \boldsymbol{W}=\left(\begin{array}{c}
\omega_{1} \\
\omega_{2} \\
\omega_{3}
\end{array}\right), \quad \boldsymbol{V}^{+}=-C_{3}^{+}\left(r^{+}-|\xi|\right)\left(\begin{array}{c}
\mathrm{i} \xi_{1} \\
\mathrm{i} \xi_{2} \\
-|\xi|
\end{array}\right), \quad \boldsymbol{V}^{-}=-C_{3}^{-}\left(r^{-}-r_{1}^{-}\right)\left(\begin{array}{c}
\mathrm{i} \xi_{1} \\
\mathrm{i} \xi_{2} \\
r_{1}^{-}
\end{array}\right) \\
& C_{3}^{+}=-\frac{1}{\left(r^{+}-|\xi|\right) P}\left\{A \left\{\left[2 \mu^{+} r^{+}+\frac{\sigma}{s} \xi^{2}\right]\left(r^{-} r_{1}^{-}-\xi^{2}\right)\right.\right. \\
& \left.+\mu^{-} r^{+}\left(r^{-2}-2 r^{-} r_{1}^{-}+\xi^{2}\right)+\rho_{0}^{-} s r^{-}\right\} \\
& +\widetilde{b}_{3}^{\prime}\left\{\mu^{+}\left(r^{-} r_{1}^{-}-\xi^{2}\right)\left(r^{+^{2}}+\xi^{2}\right)\right. \\
& \left.\left.+\mu^{-} \xi^{2}\left(r^{-2}+\xi^{2}-2 r^{-} r_{1}^{-}\right)+\rho_{0}^{-} s r^{+} r_{1}^{-}\right\}\right\}, \\
& C_{3}^{-}=-\frac{A}{P}\left\{\mu^{+}\left[r^{+}\left(r^{+}+|\xi|\right)+r^{-}\left(r^{+}-|\xi|\right)\right]+2 \mu^{-} r^{-}|\xi|+\frac{\sigma}{s}|\xi|^{3}\right\} \\
& +\frac{\widetilde{b}_{3}^{\prime}}{P}\left\{\mu^{+}\left[r^{+}|\xi|\left(r^{-}+|\xi|\right)+\left(r^{-}-|\xi|\right) \xi^{2}\right]+\mu^{-}\left(r^{-2}+\xi^{2}\right)|\xi|\right\}, \\
& \omega_{\alpha}=\frac{1}{\mu^{+} r^{+}+\mu^{-} r^{-}}\left\{\widetilde{b}_{\alpha}+\mathrm{i} \xi_{\alpha}\left[\mu^{+}\left(r^{+}-|\xi|\right) C_{3}^{+}+\mu^{-}\left(r^{-}-r_{1}^{-}\right) C_{3}^{-}\right.\right. \\
& \left.\left.+\left(\mu^{+}-\mu^{-}\right) \omega_{3}\right]\right\}, \quad \alpha=1,2, \\
& \omega_{3}=\frac{-\left(r^{+}-|\xi|\right)|\xi| C_{3}^{+}+\left(r^{-} r_{1}^{-}-\xi^{2}\right) C_{3}^{-}}{r^{+}+r^{-}} .
\end{aligned}
$$

In formulas (2.7) we have used the notation:

$$
\begin{aligned}
A= & \mathrm{i} \xi_{1} \widetilde{b}_{1}+\mathrm{i} \xi_{2} \widetilde{b}_{2}, \quad \widetilde{b}_{3}^{\prime}=\widetilde{b}_{3}+\frac{\sigma}{s} \widetilde{B}, \\
P= & \rho_{0}^{+} \mu^{+} s\left(r^{+}+|\xi|\right)\left(r^{-} r_{1}^{-}-\xi^{2}\right)+\rho_{0}^{-2} s^{2}|\xi| \\
& +\rho_{0}^{-} \mu^{+} s\left(r^{+}+|\xi|\right)\left(r^{-}|\xi|+r^{+} r_{1}^{-}+2 \xi^{2}\right) \\
& +4\left(\mu^{+}-\mu^{-}\right) \xi^{2}\left\{\mu^{+} r^{+}\left(r^{-} r_{1}^{-}-\xi^{2}\right)-\mu^{-} r^{-}\left(r^{-}-r_{1}^{-}\right)|\xi|\right\} \\
& +\frac{\sigma|\xi|^{3}}{s}\left\{\mu^{+}\left(r^{+}+|\xi|\right)\left(r^{-} r_{1}^{-}-\xi^{2}\right)+\rho_{0}^{-} r_{1}^{-} s\right\} .
\end{aligned}
$$

We observe that solution (2.6), (2.7) may be obtained by the passage to the limit $r_{1}^{+} \rightarrow|\xi|$ from a solution of the model problem with a plane interface between two compressible fluids (see [4]). 
On the other hand, it goes over as $r_{1}^{-} \rightarrow|\xi|$ into a solution of the corresponding problem for two incompressible liquids [2]. This passage may also be demonstrated in the system of equations. Let us turn $r_{1}^{-}$to $|\xi|$ that corresponds to $\beta^{-} \rightarrow \infty$, in system (2.3). In this case, the NavierStokes equations for a compressible fluid in the domain $\left\{x_{3}<0\right\}$ become the equations for the incompressible one.

We now move on to the estimates of solution (2.6), (2.7). As $C_{3}^{+}$is contained in all the expressions with multiplier $\left(r^{+}-|\xi|\right)$, which is cancelled with the denominator of $C_{3}^{+}$, the estimate of solution (2.6), (2.7) depends only on the lower bound of $P$.

We rewrite $P$ in the form:

$$
P=\sum_{j=1}^{6} I_{j}
$$

where

$$
\begin{aligned}
I_{1} & =\left(\rho_{0}^{+}+\frac{\rho_{0}^{-}|\xi|}{r_{1}^{-}}\right) s q, \\
I_{2} & =\left(\mu^{+}-\mu^{-}\right) \mu^{+} \xi^{2} r^{+}\left(r^{-} r_{1}^{-}-\xi^{2}\right), \\
I_{3} & =4 \mu^{-2} r^{-}\left(r^{-}-r_{1}^{-}\right)|\xi|^{3}, \\
I_{4} & =\mu^{+} \rho_{0}^{-} s\left(r^{+}+|\xi|\right)\left(r_{1}^{-}|\xi|+|\xi|^{3} / r^{-}\right), \\
I_{5} & =2 \mu^{+} \rho_{0}^{-} s \xi^{2}\left\{r^{+}+|\xi|-2 v^{-}|\xi| \frac{r^{-}\left(r^{-}-r_{1}^{-}\right)}{s}\right\} \\
& =2 \mu^{+} \rho_{0}^{-} s \xi^{2}\left\{r^{+}+|\xi|-2 \frac{1+\beta^{-}}{2+\beta^{-}} \frac{r^{-}|\xi|}{r^{-}+r_{1}^{-}}\right\}, \\
I_{6} & =\frac{\sigma|\xi|^{3}}{s} q, \\
q & =\mu^{+}\left(r^{+}+|\xi|\right)\left(r^{-} r_{1}^{-}-\xi^{2}\right)+\rho_{0}^{-} r_{1}^{-} s .
\end{aligned}
$$

LEMmA 2.1 For $\forall \xi \in \mathbb{R}^{2}, \forall s \in \mathbb{C}, \operatorname{Re} s>0$, the absolute value of the expression $q$ is estimated by

$$
\begin{array}{r}
|q| \geqslant \frac{\rho_{0}^{-}}{\sqrt{2}}\left|r_{1}^{-}\right||s|, \\
|q| \leqslant c_{1}\left(|s|^{3 / 2}+|s||\xi|\right) .
\end{array}
$$

Moreover, if the viscosities of the fluids satisfy the inequality

$$
v^{+}<v^{-}
$$

then $\arg (q / s) \in(0,(\arg s) / 2]$.

Proof. For definiteness, we assume from now on that $\arg s \geqslant 0$. (For $\arg s \leqslant 0$ the picture will be symmetric with respect to the axis $\operatorname{Im} s=0$.) Under this hypothesis, $\arg r^{ \pm}, \arg r_{1}^{-} \in[0, \pi / 4)$ and

$$
\arg \frac{r^{-} r_{1}^{-}-\xi^{2}}{s}=\arg \frac{1}{\left(2+\beta^{-}\right) \nu^{-}}\left(1+\frac{\left(1+\beta^{-}\right) r_{1}^{-}}{r^{-}+r_{1}^{-}}\right) \in\left(-\frac{\pi}{4}, 0\right] \text {. }
$$


Hence, $\arg q / s \in(-\pi / 4, \pi / 4)$ and

$$
|q| \geqslant|s| \operatorname{Re}\left\{\mu^{+}\left(r^{+}+|\xi|\right) \frac{r^{-} r_{1}^{-}-\xi^{2}}{s}+\rho_{0}^{-} r_{1}^{-}\right\} \geqslant \frac{\rho_{0}^{-}|s|\left|r_{1}^{-}\right|}{\sqrt{2}} .
$$

Inequality (2.10) follows from the estimate

$$
\begin{aligned}
|q| & \leqslant|s|\left\{c_{2}\left(\left|r^{+}\right|+|\xi|\right)\left(1+\sup _{s, \xi} \frac{\left|r_{1}^{-}\right|}{\left|r^{-}+r_{1}^{-}\right|}\right)+\rho_{0}^{-}\left|r_{1}^{-}\right|\right\} \\
& \leqslant c_{1}|s|\left(|s|^{1 / 2}+|\xi|\right) .
\end{aligned}
$$

Next, it follows from the inequality $v^{+}<v^{-}$that $\arg \left(r^{+}+|\xi|\right)>\arg \left(r^{-}+|\xi|\right)>$ $\arg \frac{r^{-}+r_{1}^{-}}{r_{1}^{-}}=\arg \left(r^{-}+\left|r_{1}^{-}\right|\right)$because $|\xi|<\left|r_{1}^{-}\right|$. So, $\arg q / s>0$.

It is not difficult to see that the arguments of vectors $r^{ \pm}, r_{1}^{-}, r^{ \pm}+|\xi|$ do not exceed $\frac{1}{2} \arg s$. Taking (2.11) into account again, we conclude that $\arg (q / s) \leqslant(\arg s) / 2<\pi / 4$.

Lemma 2.2 Assume that for the viscosities of the fluids the inequalities

$$
v^{+}<v^{-}, \quad \mu^{+}>\mu^{-}
$$

hold and that $\sigma \geqslant 0$. Then for $\forall \xi \in \mathbb{R}^{2}, \forall s \in \mathbb{C}, \operatorname{Re} s=\gamma>0$, we have

$$
\begin{aligned}
& |P| \geqslant c_{3}\left(\rho_{0}^{ \pm}, \nu^{-}, \beta^{-}\right) \gamma^{5 / 2}, \\
& |P| \geqslant c_{4}\left(|s|^{2}+|s|^{3 / 2}|\xi|+|s| \xi^{2}+\sigma|\xi|^{3}\right)\left(|s|^{1 / 2}+|\xi|\right) .
\end{aligned}
$$

Proof. We will show that each $\operatorname{Re}\left(I_{j} / q\right)$ is non-negative. Then for the modulus of the expression $P$, we can use the inequality

$$
|P| \geqslant|q|\left|\operatorname{Re} \sum_{j=1}^{6} \frac{I_{j}}{q}\right|=|q| \sum_{j=1}^{6} \operatorname{Re} \frac{I_{j}}{q} .
$$

We have

$$
\begin{aligned}
\operatorname{Re} \frac{I_{1}}{q}= & \operatorname{Re}\left(\rho_{0}^{+} s+\rho_{0}^{-}|\xi| \frac{s}{r_{1}^{-}}\right)=\rho_{0}^{+} \operatorname{Re} s \\
& +\rho_{0}^{-}|\xi| \frac{|s|}{\left|r_{1}^{-}\right|}\left\{\cos (\arg s) \cos \left(\arg r_{1}^{-}\right)+\sin (\arg s) \sin \left(\arg r_{1}^{-}\right)\right\} \\
\geqslant & \gamma\left(\rho_{0}^{+}+\rho_{0}^{-} \frac{|\xi|}{\sqrt{2}\left|r_{1}^{-}\right|}\right)>0
\end{aligned}
$$

because the arguments of $s$ and $r_{1}^{-}$have the same sign and consequently the product of their sines is non-negative; in addition $\cos \left(\arg r_{1}^{-}\right)>1 / \sqrt{2}$.

Next,

$$
\begin{aligned}
& \operatorname{Re} \frac{I_{2}}{q}=\operatorname{Re}\left\{4\left(\mu^{+}-\mu^{-}\right) \mu^{+} \xi^{2} \frac{r^{+}\left(r^{-} r_{1}^{-}-\xi^{2}\right)}{s} \frac{s}{q}\right\} \geqslant 0, \\
& \operatorname{Re} \frac{I_{3}}{q}=\operatorname{Re}\left\{4 \mu^{-} \frac{1+\beta^{-}}{v^{-}\left(2+\beta^{-}\right)} \frac{r^{-} \mid \xi^{3}}{r^{-}+r_{1}^{-}} \frac{s}{q}\right\} \geqslant 0
\end{aligned}
$$


as $\arg \frac{r^{+}\left(r^{-} r_{1}^{-}-\xi^{2}\right)}{s}, \arg \frac{s}{q} \in\left(-\frac{\pi}{4}, \frac{\pi}{4}\right)$, and $\arg \frac{r^{-}}{r^{-}+r_{1}^{-}} \in\left[0, \frac{\pi}{4}\right)$.

For $\frac{I_{4}}{q}$, we have

$$
\begin{aligned}
\operatorname{Re} \frac{I_{4}}{q} & =\mu^{+} \rho_{0}^{-}|\xi|\left\{\operatorname{Re} \frac{\left(r^{+}+|\xi|\right) r_{1}^{-} s}{q}+\operatorname{Re}\left(\frac{\left(r^{+}+|\xi|\right) \xi^{2}}{r_{1}^{-}} \frac{s}{q}\right)\right\} \\
& \geqslant \mu^{+} \rho_{0}^{-}|\xi| \operatorname{Re} \frac{1}{\mu^{+} \frac{r^{-} r_{1}^{-}-\xi^{2}}{s r_{1}^{-}}+\frac{\rho_{0}^{-}}{r^{+}+|\xi|}} \geqslant 0
\end{aligned}
$$

since the argument of the last fraction's denominator belongs to the interval $(-\pi / 2,0]$.

We transform $I_{5}$ in the following way:

$$
\begin{aligned}
I_{5} & =2 \mu^{+} \rho_{0}^{-} s \xi^{2}\left\{r^{+}+\frac{\left(2+\beta^{-}\right) r_{1}^{-}|\xi|-\beta^{-} r^{-}|\xi|}{\left(2+\beta^{-}\right)\left(r^{-}+r_{1}^{-}\right)}\right\} \\
& =2 \mu^{+} \rho_{0}^{-} s \xi^{2}\left\{\frac{2}{2+\beta^{-}} r^{+}+\frac{2\left(1+\beta^{-}\right) r_{1}^{-}|\xi|}{\left(2+\beta^{-}\right)\left(r^{-}+r_{1}^{-}\right)}+\frac{\beta^{-}\left(r^{+}-|\xi|\right)}{2+\beta^{-}}\right\} .
\end{aligned}
$$

Then taking the preceding arguments into account, we verify that

$$
\begin{aligned}
\operatorname{Re} \frac{I_{5}}{q} & =2 \mu^{+} \rho_{0}^{-} \xi^{2} \operatorname{Re}\left\{\frac{2}{2+\beta^{-}} \frac{r^{+}}{q / s}+\frac{2\left(1+\beta^{-}\right) r_{1}^{-}|\xi|}{\left(2+\beta^{-}\right)\left(r^{-}+r_{1}^{-}\right) q / s}\right. \\
& \left.+\frac{\beta^{-}}{\left(2+\beta^{-}\right) v^{+}} \frac{s}{\left(r^{+}+|\xi|\right) q / s}\right\}>0 .
\end{aligned}
$$

Finally,

$$
\operatorname{Re} \frac{I_{6}}{q}=\operatorname{Re} \frac{\sigma|\xi|^{3}}{s}=\frac{\sigma|\xi|^{3} \gamma}{|s|^{2}}>0
$$

Now let us return to estimate (2.14). Dropping all the terms except the first one, by virtue of (2.9) and (2.15) we obtain:

$$
|P| \geqslant \gamma \rho_{0}^{+} \frac{\rho_{0}^{-}\left|r_{1}^{-}\right||s|}{\sqrt{2}} \geqslant c_{3}\left(\rho_{0}^{ \pm}, v^{-}, \beta^{-}\right) \gamma^{5 / 2}
$$

which proves the strict positiveness of $|P|$ for $\gamma>0$. In addition, from (2.16), it follows an inequality useful for the further estimates of the absolute value of $P$. As

$$
|P| \geqslant \frac{4 \mu^{+} \rho_{0}^{-} s \xi^{2}}{2+\beta^{-}}|q| \operatorname{Re} \frac{r^{+}}{q / s}
$$

and $\arg \frac{r^{+}}{q / s} \in\left(-\frac{\pi}{4}, \frac{\pi}{4}\right)$, we have

$$
\begin{aligned}
|P| & \geqslant \frac{4 \mu^{+} \rho_{0}^{-} \xi^{2}}{2+\beta^{-}} \frac{\left|r^{+}\right||s|}{\sqrt{2}} \\
& =c_{5}|s|\left|r^{+}\right| \xi^{2} \geqslant c_{6}\left(|s|^{3 / 2} \xi^{2}+|s||\xi|^{3}\right) .
\end{aligned}
$$

In order to prove the second inequality in (2.13), we consider two cases. 
Case 1. Let $|s|<\xi^{2}$. Then

$$
|s|^{5 / 2}+|s|^{2}|\xi| \leqslant|s|^{3 / 2} \xi^{2}+|s||\xi|^{3} \leqslant \frac{|P|}{c_{6}} .
$$

In view of formula (2.8) for $P$, we have

$$
\sigma|\xi|^{3}\left|\frac{q}{s}\right| \leqslant|P|+\sum_{j=1}^{5}\left|I_{j}\right| .
$$

Let us estimate $\left|I_{j}\right|, \quad j=1, \ldots, 5$. Using (2.10), we obtain:

$$
\begin{aligned}
\left|I_{1}\right| & \leqslant\left(\rho_{0}^{+}+\frac{\rho_{0}^{-}|\xi|}{\left|r_{1}^{-}\right|}\right)|s||q| \leqslant c_{7}\left(|s|^{5 / 2}+|s|^{2}|\xi|\right) \leqslant \frac{c_{7}}{c_{6}}|P|, \\
\left|I_{2}\right| & \leqslant \frac{4\left(\mu^{+}-\mu^{-}\right) \mu^{+}}{\left(2+\beta^{-}\right) v^{-}}|s|\left|r^{+}\right| \xi^{2} \max _{s, \xi}\left|1+\frac{\left(1+\beta^{-}\right) r_{1}^{-}}{r^{-}+r_{1}^{-}}\right| \leqslant c_{8}|s|\left|r^{+}\right| \xi^{2} \\
& \leqslant \frac{c_{8}}{c_{5}}|P|, \\
\left|I_{3}\right| & \leqslant c_{9} \max _{s, \xi}\left|\frac{r^{-}}{r^{-}+r_{1}^{-}}\right||s||\xi|^{3} \leqslant \frac{c_{9}}{c_{6}}|P|, \\
\left|I_{4}\right| & \leqslant c_{10}|s|\left(\left|r^{+}\right|+|\xi|\right)\left(\left|r_{1}^{-}\right||\xi|+\xi^{2}\right) \leqslant c_{11}\left(|s|^{2}|\xi|+|s||\xi|^{3}\right) \leqslant \frac{c_{11}}{c_{6}}|P|, \\
\left|I_{5}\right| & \leqslant c_{12}|s| \xi^{2}\left(\left|r^{+}\right|+|\xi|\right) \leqslant c_{12}\left(\frac{1}{c_{5}}+\frac{1}{c_{6}}\right)|P| .
\end{aligned}
$$

Hence,

$$
\sigma|\xi|^{4} \leqslant \sigma|\xi|^{3}\left|r_{1}^{-}\right| \leqslant \sigma \frac{\sqrt{2}}{\rho_{0}^{-}}|\xi|^{3}\left|\frac{q}{s}\right| \leqslant c_{13}|P| .
$$

Thus, (2.13) is proved for the first case.

Case 2. Now consider $|s| \geqslant \xi^{2}$. From (2.17) it follows that

$$
\sigma|\xi|^{3}\left(|s|^{1 / 2}+|\xi|\right) \leqslant 2 \sigma \frac{|s||\xi|^{3}}{\sqrt{\gamma}} \leqslant \frac{2 \sigma|P|}{c_{6} \sqrt{\gamma}} .
$$

We gather all terms in $P$ of orders $|s|^{5 / 2}$ and $|s|^{2}|\xi|$ and introduce the function

$$
I_{0}=I_{1}+\mu^{+} \rho_{0}^{-} s\left(r^{+}-|\xi|\right) r_{1}^{-}|\xi| .
$$

Evaluating its absolute value by its real part, we deduce the estimate

$$
\begin{aligned}
\left|I_{0}\right| & \geqslant|s|^{2} \operatorname{Re}\left\{\rho_{0}^{+} \frac{q}{s}+\frac{\rho_{0}^{-}|\xi|}{r_{1}^{-}} \frac{q}{s}+\rho_{0}^{+} \rho_{0}^{-} \frac{r_{1}^{-}|\xi|}{r^{+}+|\xi|}\right\} \\
& \geqslant \frac{1}{\sqrt{2}}|s|^{2}\left\{\frac{\rho_{0}^{+} \rho_{0}^{-}}{\sqrt{2}}\left|r_{1}^{-}\right|+\frac{\rho_{0}^{-2}}{\sqrt{2}}|\xi|+\rho_{0}^{+} \rho_{0}^{-} \min _{s, \xi}\left|\frac{r_{1}^{-}}{r^{+}+|\xi|}\right| \xi \mid\right\} .
\end{aligned}
$$


We note that in the last inequality we have used Lemma 2.1 and the fact that the argument of each term lies in the interval $(-\pi / 4, \pi / 4)$.

At last, with regard to (2.17), (2.18) we have:

$$
\begin{aligned}
c_{14}\left(|s|^{5 / 2}+|s|^{2}|\xi|\right) \leqslant\left|I_{0}\right| \leqslant|P| & +\sum_{j=2, j \neq 4}^{6}\left|I_{j}\right|+2 \mu^{+} \rho_{0}^{-}|s|\left|r_{1}^{-}\right| \xi^{2} \\
& +\mu^{+} \rho_{0}^{-}|s| \frac{\left|r^{+}+\right| \xi||}{\left|r_{1}^{-}\right|}|\xi|^{3} \leqslant c_{15}|P| .
\end{aligned}
$$

So, (2.13) is completely proved.

Now we may estimate the solution (2.6) easily.

LEMMA 2.3 For the coefficients $\boldsymbol{W}, \boldsymbol{V}^{ \pm}$defined by formulas (2.7), the inequalities

$$
\begin{aligned}
& \left|\boldsymbol{V}^{ \pm}\right| \leqslant c_{16}(\gamma)\left(\sum_{\alpha=1}^{2}\left|\tilde{b_{\alpha}}\right|+\frac{\left|\widetilde{b_{3}}\right||\xi|+\sigma|\widetilde{B}|}{\sqrt{|s|+\xi^{2}}}\right), \\
& |\boldsymbol{W}| \leqslant c_{17}\left(\sum_{\alpha=1}^{2} \frac{\left|\widetilde{b_{\alpha}}\right|}{\sqrt{|s|+\xi^{2}}}+\frac{\left|\widetilde{b_{3}}\right||\xi|+\sigma|\widetilde{B}|}{|s|+\xi^{2}}\right)
\end{aligned}
$$

hold.

Proof. Taking into account the evident inequality

$$
\frac{\left|r^{-} r_{1}^{-}-\xi^{2}\right|}{|s|} \leqslant \frac{1}{(2+\beta) v^{-}}\left(1+(1+\beta) \sup _{s, \xi} \frac{\left|r_{1}^{-}\right|}{\left|r^{-}+r_{1}^{-}\right|}\right) \leqslant c_{18}\left(v^{-}, \beta^{-}\right),
$$

we obtain

$$
\begin{aligned}
\left|\boldsymbol{V}^{+}\right| \leqslant & c_{19} \sum_{\alpha=1}^{2} \frac{\left|\widetilde{b_{\alpha}}\right| \xi^{2}}{|P|}|s|\left(\left|r^{+}\right|+\left|r^{-}\right|\right) \\
& +c_{20} \frac{\left(\left|\tilde{b_{3}}\right||s|+\sigma|\widetilde{B}|\right)}{|P|}|\xi|\left\{|s|+\xi^{2}+\left|r^{+}\right|\left|r_{1}^{-}\right|\right\} \\
\left|\boldsymbol{V}^{-}\right| \leqslant & \frac{\left(1+\beta^{-}\right)|s|\left|r_{1}^{-}\right|}{\left(2+\beta^{-}\right) v^{-}\left|r^{-}+r_{1}^{-}\right|}\left|C_{3}^{-}\right| \\
\leqslant & \frac{c_{21}|s|}{|P|}\left\{\sum_{\alpha=1}^{2}\left|\widetilde{b_{\alpha}}\right||\xi|\left[|s|+\xi^{2}+\left(\left|r^{+}\right|+\left|r_{1}^{-}\right|\right)|\xi|+\frac{\sigma|\xi|^{3}}{|s|}\right]\right. \\
& \left.+\left(\left|\tilde{b}_{3}\right|+\frac{\sigma|\widetilde{B}|}{|s|}\right)|\xi|\left[|s|+\xi^{2}+\left|r^{+}\right||\xi|\right]\right\} .
\end{aligned}
$$


From here, in view of (2.13), we obtain the estimate

$$
\left|\boldsymbol{V}^{ \pm}\right| \leqslant c_{22}\left\{\sum_{\alpha=1}^{2}\left|\tilde{b_{\alpha}}\right|+\frac{\left|\widetilde{b_{3}}\right||\xi|+\sigma|\widetilde{B}|\left(\frac{1}{\sigma}+\frac{1}{\sqrt{\gamma}}\right)}{|s|^{1 / 2}+|\xi|}\right\}
$$

(the term with $|\widetilde{B}|$ drops out for $\sigma=0$ ).

The inequality (2.20) follows from the relations

$$
\begin{aligned}
&\left|\omega_{3}\right| \leqslant \frac{c_{23}}{\left|r^{+}+r^{-}\right|}\left(\left|\boldsymbol{V}^{+}\right|+c_{18}|s|\left|C_{3}^{-}\right|\right) \\
&\left|\omega_{\alpha}\right| \leqslant \frac{1}{\left|\mu^{+} r^{+}+\mu^{-} r^{-}\right|}\left(\left|\widetilde{b_{\alpha}}\right|+\mu^{+}\left|\boldsymbol{V}^{+}\right|+\mu^{-}\left|\boldsymbol{V}^{-}\right|+\left(\mu^{+}-\mu^{-}\right)\left|\omega_{3}\right||\xi|\right), \\
& \alpha=1,2,
\end{aligned}
$$

and from estimates (2.19), (2.21).

In the dual Fourier-Laplace space, we introduce normalizations corresponding to the norms defined in Section 1. It is possible to do so by means of a Parceval equality for the transform (2.2)

$$
\int_{-\infty}^{\infty} \int_{\mathbb{R}^{2}}\left|\tilde{f}\left(\xi, x_{3}, \gamma+\mathrm{i} \xi_{0}\right)\right|^{2} \mathrm{~d} \xi \mathrm{d} \xi_{0}=(2 \pi)^{3} \int_{0}^{\infty} \mathrm{e}^{-2 \gamma t} \int_{\mathbb{R}^{2}}|f(x, t)|^{2} \mathrm{~d} x^{\prime} \mathrm{d} t .
$$

Thus in Section 2 of [7] it was shown that for $\forall \gamma \geqslant 0$ the norm $\|u\|_{\bigcup_{i} H_{\gamma}^{m, m / 2}\left(D_{\infty}^{i}\right)}$ is equivalent to the norm whose square is given by

$$
\begin{array}{r}
\||u|\|_{m, \gamma, D_{\infty}^{3}}^{2} \equiv \int_{\mathbb{R}^{2}} \int_{-\infty}^{\infty}\left\{\sum_{j<m}\left\|\frac{\partial^{j}}{\partial x_{3}^{j}} \widetilde{u}\left(\xi, x_{3}, s\right)\right\|_{\mathbb{R}_{-} \cup \mathbb{R}_{+}}^{2}|r|^{2 m-2 j}\right. \\
\left.+\|\widetilde{u}(\xi, \cdot, s)\|_{\dot{W}_{2}^{m}}^{2}\left(\mathbb{R}_{-}\right) \cup \dot{W}_{2}^{m}\left(\mathbb{R}_{+}\right)\right\} \mathrm{d} \xi_{0} \mathrm{~d} \xi
\end{array}
$$

where $s=\gamma+\mathrm{i} \xi_{0}, r=\sqrt{s+\xi^{2}}, \mathbb{R}_{ \pm}=\{y \in \mathbb{R} \mid \pm y>0\}, D_{\infty}^{3}=D_{\infty}^{-} \cup D_{\infty}^{+}$.

For the trace of a function $u$ on the plane $\mathbb{R}^{2}$, the norm $\|u\|_{H_{\gamma}^{m, m / 2}\left(\mathbb{R}_{\infty}^{2}\right)}$ is equivalent to the norm

$$
\||u|\|_{m, \gamma, \mathbb{R}_{\infty}^{2}} \equiv\left(\int_{\mathbb{R}^{2}} \int_{-\infty}^{\infty}|\widetilde{u}(\xi, 0, s)|^{2}|r|^{2 m} \mathrm{~d} \xi_{0} \mathrm{~d} \xi\right)^{1 / 2}
$$

while the norm $\|u\|_{H_{\gamma}^{m+1 / 2,1 / 2, m / 2}\left(\mathbb{R}_{\infty}^{2}\right)}$ is equivalent to the norm

$$
|u|_{m, \gamma, \mathbb{R}_{\infty}^{2}} \equiv\left(\int_{\mathbb{R}^{2}}|\xi| \int_{-\infty}^{\infty}|\widetilde{u}(\xi, 0, s)|^{2}|r|^{2 m} \mathrm{~d} \xi_{0} \mathrm{~d} \xi\right)^{1 / 2}
$$

Consider now the velocity vector $\widetilde{\boldsymbol{v}}$ from (2.6). We can write:

$$
\left|\frac{\mathrm{d}^{j} \widetilde{\boldsymbol{v}}\left(x_{3}\right)}{\mathrm{d} x_{3}^{j}}\right| \leqslant c_{24}\left(|\boldsymbol{W}|\left|\frac{\mathrm{d}^{j} e_{0}^{ \pm}\left(x_{3}\right)}{\mathrm{d} x_{3}^{j}}\right|+\left|\boldsymbol{V}^{ \pm}\right|\left|\frac{\mathrm{d}^{j} e_{1}^{ \pm}\left(x_{3}\right)}{\mathrm{d} x_{3}^{j}}\right|\right), \quad \pm x_{3}>0,
$$


where $j=0,1, \ldots$ We estimate the derivatives of functions $e_{0}^{ \pm}, e_{1}^{ \pm}$according to the inequalities (see Lemma 3.1 from [7]):

$$
\begin{aligned}
& \pm \int_{0}^{ \pm \infty}\left|\frac{\mathrm{d}^{j} e_{0}^{ \pm}\left(x_{3}\right)}{\mathrm{d} x_{3}^{j}}\right|^{2} \mathrm{~d} x_{3} \leqslant \frac{\left|r^{ \pm}\right|^{2 j-1}}{\sqrt{2}}, \\
& \pm \int_{0}^{ \pm \infty} \int_{0}^{\infty}\left|\frac{\mathrm{d}^{j} e_{0}^{ \pm}\left(x_{3} \pm z\right)}{\mathrm{d} x_{3}^{j}}-\frac{\mathrm{d}^{j} e_{0}^{ \pm}\left(x_{3}\right)}{\mathrm{d} x_{3}^{j}}\right|^{2} \frac{\mathrm{d} z \mathrm{~d} x_{3}}{z^{1+2 \alpha}} \leqslant c_{26}^{ \pm}|r|^{2(j+\alpha)-1} \\
& \pm \int_{0}^{ \pm \infty}\left|\frac{\mathrm{d}^{j} e_{1}^{ \pm}\left(x_{3}\right)}{\mathrm{d} x_{3}^{j}}\right|^{2} \mathrm{~d} x_{3} \leqslant c_{27}^{ \pm}|r|^{2 j-3} \\
& \pm \int_{0}^{ \pm \infty} \int_{0}^{\infty}\left|\frac{\mathrm{d}^{j} e_{1}^{ \pm}\left(x_{3} \pm z\right)}{\mathrm{d} x_{3}^{j}}-\frac{\mathrm{d}^{j} e_{1}^{ \pm}\left(x_{3}\right)}{\mathrm{d} x_{3}^{j}}\right|^{2} \frac{\mathrm{d} z \mathrm{~d} x_{3}}{z^{1+2 \alpha}} \leqslant c_{28}^{ \pm}|r|^{2(j+\alpha)-3}
\end{aligned}
$$

where $j=0,1, \ldots, \alpha \in(0,1), r=\sqrt{s+\xi^{2}}, s=\gamma+\mathrm{i} \xi_{0}, \gamma>0, \xi \in \mathbb{R}^{2}$, and the constants $c_{26}^{ \pm}, c_{27}^{ \pm}, c_{28}^{ \pm}$do not depend on $|r|$. Then for the norm of vector $v$ the relation

$$
\||\boldsymbol{v}|\|_{2+l, \gamma, D_{\infty}^{3}}^{2} \leqslant c_{29} \int_{\mathbb{R}^{2}} \int_{-\infty}^{\infty}\left(|\boldsymbol{W}|^{2}|r|^{2 l+3}+\left|\boldsymbol{V}^{ \pm}\right|^{2}|r|^{2 l+1}\right) \mathrm{d} \xi_{0} \mathrm{~d} \xi
$$

holds.

As the function $\mathrm{e}^{-|\xi| x_{3}}$ satisfies inequalities (2.22) with $|r|$ replaced by $|\xi|$, for the pressure gradient

$$
\nabla \widetilde{p}=\mu^{+} \boldsymbol{V}^{+}\left(r^{+}+|\xi|\right) \mathrm{e}^{-|\xi| x_{3}}, \quad x_{3}>0
$$

the inequality

$$
\||\nabla p|\|_{l, \gamma, D_{\infty}^{+}}^{2} \leqslant c_{30} \int_{\mathbb{R}^{2}} \int_{-\infty}^{\infty}\left|V^{+}\right|^{2}\left|r^{+}\right|^{2}|\xi|^{2 l-1} \mathrm{~d} \xi_{0} \mathrm{~d} \xi
$$

holds.

And finally, as a consequence of Lemma 2.3 we conclude that

$$
\begin{aligned}
& \||\boldsymbol{v}|\|_{2+l, \gamma, D_{\infty}^{3}}+\||\nabla p|\|_{l, \gamma, D_{\infty}^{+}} \\
& \leqslant c_{31}\left(\int_{\mathbb{R}^{2}} \int_{-\infty}^{\infty}\left(\sum_{\alpha=1}^{2}\left|\widetilde{b_{\alpha}}\right|^{2}+\frac{\left|\widetilde{b_{3}}\right|^{2}|\xi|^{2}+\sigma^{2}|\widetilde{B}|^{2}}{|r|^{2}}\right)|r|^{2 l+1} \mathrm{~d} \xi_{0} \mathrm{~d} \xi\right)^{1 / 2} \\
& \leqslant c_{32}\left\{\sum_{\alpha=1}^{2}\left\|\left|b_{\alpha}\right|\right\|_{l+1 / 2, \gamma, \mathbb{R}_{\infty}^{2}}+\left|b_{3}\right|_{l, \gamma, \mathbb{R}_{\infty}^{2}}+\sigma\||B|\|_{l-1 / 2, \gamma, \mathbb{R}_{\infty}^{2}}\right\} .
\end{aligned}
$$

Thus, we have proved the unique solvability of problem (2.1).

THEOREM 2.4 Assume that $l>1 / 2, \gamma \geqslant \gamma_{0}>0, \sigma \geqslant 0$ and that inequalities (2.12) hold for the viscosities of the liquids. In addition, we suppose that $b_{\alpha} \in H_{\gamma}^{l+1 / 2, l / 2+1 / 4}\left(\mathbb{R}_{\infty}^{2}\right), \alpha=1,2, b_{3} \in$ $H_{\gamma}^{l+1 / 2,1 / 2, l / 2}\left(\mathbb{R}_{\infty}^{2}\right)$, and $B \in H_{\gamma}^{l-1 / 2, l / 2-1 / 4}\left(\mathbb{R}_{\infty}^{2}\right)$.

Then there exists a unique solution $(\boldsymbol{v}, p)$ of problem (2.1) such that $\boldsymbol{v} \in$ $\bigcup_{i=-,+} \boldsymbol{H}_{\gamma}^{2+l, 1+l / 2}\left(D_{\infty}^{i}\right), \nabla p \in \boldsymbol{H}_{\gamma}^{l, l / 2}\left(D_{\infty}^{+}\right)$, and inequality (2.25) holds. 
REMARK 2.5 It should be noted that the uniqueness of solution (2.6), (2.7) for $\gamma \geqslant \gamma_{0}>0$ is guaranteed by the first inequality in (2.13), i.e. by the fact that in this case $P$ is separated from zero.

\section{The non-homogeneous model problem}

In this section we will prove the unique solvability of the non-homogeneous problem with plane interface between the fluids by reducing it to problem (2.1).

Let $T \leqslant \infty$ and consider the system

$$
\begin{gathered}
\mathcal{D}_{t} \boldsymbol{w}-v^{+} \nabla^{2} \boldsymbol{w}+\frac{1}{\rho_{0}^{+}} \nabla q=\boldsymbol{f}, \quad \nabla \cdot \boldsymbol{w}=g \quad \text { in } \quad D_{T}^{+}=\mathbb{R}_{+}^{3} \times(0, T), \\
\mathcal{D}_{t} \boldsymbol{w}-v^{-} \nabla^{2} \boldsymbol{w}-\left(v^{-}+\kappa^{-}\right) \nabla(\nabla \cdot \boldsymbol{w})=\boldsymbol{f} \quad \text { in } \quad D_{T}^{-}=\mathbb{R}_{-}^{3} \times(0, T), \\
\left.\boldsymbol{w}\right|_{t=0}=0, \quad \boldsymbol{w} \underset{|x| \rightarrow \infty}{\longrightarrow} 0, \quad q \underset{|x| \rightarrow \infty}{\longrightarrow} 0,\left.\quad[\boldsymbol{w}]\right|_{x_{3}=0}=0, \\
-\left.\left[\mu^{ \pm}\left(\frac{\partial w_{\alpha}}{\partial x_{3}}+\frac{\partial w_{3}}{\partial x_{\alpha}}\right)\right]\right|_{x_{3}=0}=a_{\alpha}\left(x^{\prime}, t\right), x^{\prime}=\left(x_{1}, x_{2}\right), t \in(0, T), \alpha=1,2 \\
-\left.\left(q+\lambda^{-} \nabla \cdot \boldsymbol{w}\right)\right|_{x_{3}=0}+\left.\left[2 \mu^{ \pm} \frac{\partial w_{3}}{\partial x_{3}}\right]\right|_{x_{3}=0}+\left.\sigma \Delta^{\prime} \int_{0}^{t} w_{3}\right|_{x_{3}=0} \mathrm{~d} \tau \\
=a_{3}+\sigma \int_{0}^{t} A \mathrm{~d} \tau \quad \text { on } \mathbb{R}_{T}^{2} \equiv \mathbb{R}^{2} \times(0, T) .
\end{gathered}
$$

Theorem 3.1 Let $T>0, \gamma \geqslant \gamma_{0}>0, \sigma \geqslant 0$, and let the viscosities of the liquids $\mu^{ \pm}, \nu^{ \pm}$ satisfy inequalities (2.12). In addition, we assume that, for some $l>1 / 2, \boldsymbol{f} \in \bigcup_{i=-,+} \boldsymbol{H}_{\gamma}^{l, l / 2}\left(D_{T}^{i}\right)$, $g \in H_{\gamma}^{1+l, 1 / 2+l / 2}\left(D_{T}^{+}\right), g=\nabla \cdot \boldsymbol{R}, \boldsymbol{R} \in H_{\gamma}^{0, l+1 / 2}\left(D_{T}^{+}\right), a_{\alpha} \in H_{\gamma}^{l+1 / 2, l / 2+1 / 4}\left(\mathbb{R}_{T}^{2}\right), \alpha=1,2$, $a_{3} \in H_{\gamma}^{l+1 / 2,1 / 2, l / 2}\left(\mathbb{R}_{T}^{2}\right)$, and $A \in H_{\gamma}^{l-1 / 2, l / 2-1 / 4}\left(\mathbb{R}_{T}^{2}\right)$. Then problem (3.1) is uniquely solvable on the interval $(0, T]$ and for its solution $(\boldsymbol{w}, q), \boldsymbol{w} \in \bigcup_{i=-,+} \boldsymbol{H}_{\gamma}^{2+l, 1+l / 2}\left(D_{T}^{i}\right), \nabla q \in \boldsymbol{H}_{\gamma}^{l, l / 2}\left(D_{T}^{+}\right)$, the inequality

$$
\begin{aligned}
& \|\boldsymbol{w}\|_{\bigcup_{i} \boldsymbol{H}_{\gamma}^{2+l, 1+l / 2}\left(D_{T}^{i}\right)}+\|\nabla q\|_{\boldsymbol{H}_{\gamma}^{l, l / 2}\left(D_{T}^{+}\right)} \leqslant c_{1}\left\{\|\boldsymbol{f}\|_{\bigcup_{i} \boldsymbol{H}_{\gamma}^{l, l / 2}\left(D_{T}^{i}\right)}\right. \\
& +\|g\|_{H_{\gamma}^{1+l, 1 / 2+l / 2}\left(D_{T}^{+}\right)}+\|\boldsymbol{R}\|_{\boldsymbol{H}_{\gamma}^{0,1+l / 2}\left(D_{T}^{+}\right)}+\sum_{\alpha=1}^{2}\left\|a_{\alpha}\right\|_{H_{\gamma}^{l+1 / 2, l / 2+1 / 4}\left(\mathbb{R}_{T}^{2}\right)} \\
& \left.+\left\|a_{3}\right\|_{H_{\gamma}^{l+1 / 2,1 / 2, l / 2}\left(\mathbb{R}_{T}^{2}\right)}+\sigma\|A\|_{H_{\gamma}^{l-1 / 2, l / 2-1 / 4}\left(\mathbb{R}_{T}^{2}\right)}\right\}
\end{aligned}
$$

holds.

Proof. We shall look for the velocity vector $\boldsymbol{w}$ in the form: $\boldsymbol{w}=\boldsymbol{u}+\boldsymbol{w}^{\prime}+\boldsymbol{v}^{\prime}$ where

$$
\begin{aligned}
& \boldsymbol{u}= \begin{cases}\boldsymbol{u}_{1}^{+}, & x_{3}>0, \\
\boldsymbol{u}_{1}^{-}+\boldsymbol{u}_{2}^{-}, & x_{3}<0,\end{cases} \\
& \boldsymbol{w}^{\prime}= \begin{cases}\nabla \Phi, & x_{3}>0, \\
0, & x_{3}<0,\end{cases}
\end{aligned}
$$


$\Phi(x, t)$ being a solution of the Dirichlet problem for the Poisson equation

$$
\begin{aligned}
\Delta \Phi(x, t) & =g-\nabla \cdot \boldsymbol{u}_{1}^{+} \equiv g^{\prime}(x, t), \quad x \in \mathbb{R}_{+}^{3}, \quad t>0, \\
\left.\Phi\right|_{x_{3}=0} & =0 .
\end{aligned}
$$

Next, let $\left(\boldsymbol{v}^{\prime}, p^{\prime}\right)$ be a solution of the homogeneous problem (2.1) with the boundary functions

$$
\begin{aligned}
b_{\alpha} & =a_{\alpha}+\left.\left[\mu^{ \pm}\left(\frac{\partial u_{\alpha}}{\partial x_{3}}+\frac{\partial u_{3}}{\partial x_{\alpha}}\right)\right]\right|_{x_{3}=0}+\left.\mu^{+}\left(\frac{\partial w_{\alpha}^{\prime}}{\partial x_{3}}+\frac{\partial w_{3}^{\prime}}{\partial x_{\alpha}}\right)\right|_{x_{3}=0}, \quad \alpha=1,2, \\
b_{3} & =a_{3}-\left[2 \mu^{ \pm} \frac{\partial\left(u_{3}+w_{3}^{\prime}\right)}{\partial x_{3}}\right]\left|+\lambda^{-} \nabla \cdot\left(\boldsymbol{u}_{1}^{-}+\boldsymbol{u}_{2}^{-}\right)\right|_{x_{3}=0}+\left.\mu^{+} g^{\prime}\right|_{x_{3}=0}, \\
B & =A-\left.\Delta^{\prime}\left(u_{3}+w_{3}^{\prime}\right)\right|_{x_{3}=0}
\end{aligned}
$$

then as a pressure function associated with $\boldsymbol{w}$ we can take $q=p^{\prime}+\rho_{0}^{+}\left(v^{+} g^{\prime}-\partial \Phi / \partial t\right)(\Phi=0$ in $\left.\mathbb{R}_{-}^{3}\right)$.

We define the vector-function $\boldsymbol{u}$ as follows. $\boldsymbol{u}_{1}^{+}$is a solution of the Cauchy problem for the heat equation

$$
\begin{aligned}
\mathcal{D}_{t} \boldsymbol{u}_{1}^{+}-v^{+} \Delta \boldsymbol{u}_{1}^{+} & =\boldsymbol{f}^{+} \quad \text { in } \mathbb{R}_{T}^{3} \equiv \mathbb{R}^{3} \times(0, T), \\
\left.\boldsymbol{u}_{1}^{+}\right|_{t=0} & =0
\end{aligned}
$$

$\boldsymbol{u}_{1}^{-}$is a solution of the Cauchy problem too:

$$
\begin{gathered}
\mathcal{D}_{t} \boldsymbol{u}_{1}^{-}-v^{-} \Delta \boldsymbol{u}_{1}^{-}-\left(v^{-}+\kappa^{-}\right) \nabla\left(\nabla \cdot \boldsymbol{u}_{1}^{-}\right)=\boldsymbol{f}^{-} \quad \text { in } \mathbb{R}_{T}^{3}, \\
\left.\boldsymbol{u}_{1}^{-}\right|_{t=0}=0 .
\end{gathered}
$$

(Here $\boldsymbol{f}^{+}$and $\boldsymbol{f}^{-}$are continuous extensions of restrictions $\left.\boldsymbol{f}\right|_{x_{3}>0}$ and $\left.\boldsymbol{f}\right|_{x_{3}<0}$, respectively, on the whole $\mathbb{R}_{\infty}^{3}$.) We determine $\boldsymbol{u}_{2}^{-}$as a solution of the initial-boundary value problem

$$
\begin{gathered}
\mathcal{D}_{t} \boldsymbol{u}_{2}^{-}-v^{-} \Delta \boldsymbol{u}_{2}^{-}-\left(v^{-}+\kappa^{-}\right) \nabla\left(\nabla \cdot \boldsymbol{u}_{2}^{-}\right)=0 \quad \text { in } D_{T}^{-}, \\
\left.\boldsymbol{u}_{2}^{-}\right|_{t=0}=0,\left.\quad \boldsymbol{u}_{2}^{-}\right|_{x_{3}=0}=\left.\left(\boldsymbol{u}_{1}^{+}-\boldsymbol{u}_{1}^{-}-\boldsymbol{w}^{\prime}\right)\right|_{x_{3}=0} \equiv \boldsymbol{\varphi} .
\end{gathered}
$$

Now, we show that every function introduced above is well defined and that its norm is bounded by the norms of the data of problem (3.1).

We consider $T=\infty$ because every given function admits an extension to $t>T$ in the same class.

Let us take the Laplace transform on $t$ and the Fourier transform on the all spatial variables in problems (3.5), (3.6). Then it is easy to see that for their solutions the estimate

$$
\int_{-\infty}^{\infty} \int_{\mathbb{R}^{3}}\left|\widetilde{\boldsymbol{u}_{1}^{ \pm}}(\xi, s)\right|^{2}\left(|s|+\xi^{2}\right)^{l+2} \mathrm{~d} \xi \mathrm{d} \xi_{0} \leqslant c_{2} \int_{-\infty}^{\infty} \int_{\mathbb{R}^{3}}\left|\widetilde{\boldsymbol{f}^{ \pm}}(\xi, s)\right|^{2}\left(|s|+\xi^{2}\right)^{l} \mathrm{~d} \xi \mathrm{d} \xi_{0}
$$

holds. Here $\xi=\left(\xi_{1}, \xi_{2}, \xi_{3}\right), \xi^{2}=\xi_{1}^{2}+\xi_{2}^{2}+\xi_{3}^{2}, \quad s=\gamma+\mathrm{i} \xi_{0}$. Estimate (3.8) is equivalent to the inequalities

$$
\left\|\boldsymbol{u}_{1}^{ \pm}\right\|_{\boldsymbol{H}_{\gamma}^{2+l, 1+l / 2}\left(\mathbb{R}_{\infty}^{3}\right)} \leqslant c_{3}\left\|\boldsymbol{f}^{ \pm}\right\|_{\boldsymbol{H}_{\gamma}^{l, l / 2}\left(\mathbb{R}_{\infty}^{3}\right)} \leqslant c_{4}^{ \pm}\|\boldsymbol{f}\|_{\bigcup_{i} \boldsymbol{H}_{\gamma}^{l, l / 2}\left(D_{\infty}^{i}\right)}
$$


After the partial Fourier-Laplace transform (2.2), a solution of system (3.7) may be expressed in form (2.6):

$$
\widetilde{\boldsymbol{u}_{2}^{-}}=\boldsymbol{W} e_{0}^{-}+\boldsymbol{V} e_{1}^{-}
$$

It is not difficult to show that

$$
\boldsymbol{W}=\tilde{\varphi}, \quad \boldsymbol{V}=-C_{3}\left(r^{-}-r_{1}^{-}\right)\left(\begin{array}{l}
\mathrm{i} \xi_{1} \\
\mathrm{i} \xi_{2} \\
r_{1}^{-}
\end{array}\right)
$$

where $C_{3}=\frac{\mathrm{i} \xi_{1} \widetilde{\varphi}_{1}+\mathrm{i} \xi_{2} \widetilde{\varphi}_{2}+r^{-} \widetilde{\varphi}_{3}}{r^{-} r_{1}^{-}-\xi^{2}}$. Taking inequalities (2.22)-(2.24) into consideration, we conclude that

$$
\begin{aligned}
\left\|\left|\boldsymbol{u}_{2}^{-}\right|\right\|_{2+l, \gamma, D_{\infty}^{-}} & \leqslant c_{5}\||\boldsymbol{\varphi}|\|_{3 / 2+l, \gamma, \mathbb{R}_{\infty}^{2}} \\
& \leqslant c_{5}\left(\left\|\left|\boldsymbol{u}_{1}^{+}\right|\right\|_{2+l, \gamma, D_{\infty}^{+}}+\left\|\left|\boldsymbol{u}_{1}^{-}\right|\right\|_{2+l, \gamma, D_{\infty}^{-}}+\left\|\left|\boldsymbol{w}^{\prime}\right|\right\|_{2+l, \gamma, D_{\infty}^{+}}\right) .
\end{aligned}
$$

Thus, it remains to estimate only the norm of $\boldsymbol{w}^{\prime}=\nabla \Phi$. To this end, we turn our attention to problem (3.3). By means of the Green formula for the Poisson equation in the half-space

$$
\Phi(x, t)=\int_{\mathbb{R}_{+}^{3}} G(x, y) g^{\prime}(y, t) \mathrm{d} y=-\int_{\mathbb{R}_{+}^{3}} \nabla_{y} G(x, y) \cdot\left(\boldsymbol{R}(y, t)-\boldsymbol{u}_{1}^{+}(y, t)\right) \mathrm{d} y,
$$

where $G(x, y)=-\frac{1}{4 \pi}\left(\frac{1}{|x-y|}-\frac{1}{|x-y *|}\right), \quad y *=\left(y_{1}, y_{2},-y_{3}\right)$, it was proved in [7] that

$$
\begin{aligned}
\left\|\boldsymbol{w}^{\prime}\right\|_{\boldsymbol{H}_{\gamma}^{2+l, 1+l / 2}\left(D_{T}^{+}\right)} \leqslant c_{6}\left\{\|g\|_{H_{\gamma}^{1+l, 0}\left(D_{T}^{+}\right)}\right. & +\|\boldsymbol{R}\|_{\boldsymbol{H}_{\gamma}^{0,1+l / 2}\left(D_{T}^{+}\right)} \\
& \left.+\left\|\boldsymbol{u}_{1}^{+}\right\|_{\boldsymbol{H}_{\gamma}^{2+l, 1+l / 2}\left(D_{T}^{+}\right)}\right\} .
\end{aligned}
$$

Finally, the evalution of the solution of the initial-boundary value problem (2.1), (3.4) is given by Theorem 2.4. According to inequality (2.25), we have:

$$
\begin{aligned}
& \left\|\left|\boldsymbol{v}^{\prime}\right|\right\|_{2+l, \gamma, D_{\infty}^{3}}+\left\|\left|\nabla p^{\prime}\right|\right\|_{l, \gamma, D_{\infty}^{+}} \leqslant c_{7}\left\{\sum_{\alpha=1}^{2}\left\|\left|a_{\alpha}\right|\right\|_{l+1 / 2, \gamma, \mathbb{R}_{\infty}^{2}}+\left|a_{3}\right|_{l, \gamma, \mathbb{R}_{\infty}^{2}}\right. \\
& \left.+\sigma\||A|\|_{l-1 / 2, \gamma, \mathbb{R}_{\infty}^{2}}+\left\|\left|\boldsymbol{u}+\boldsymbol{w}^{\prime}\right|\right\|_{l+3 / 2, \gamma, \mathbb{R}_{\infty}^{2}}+\left|g^{\prime}\right|_{l, \gamma, \mathbb{R}_{\infty}^{2}}\right\} \\
& \leqslant c_{7}\left\{\sum_{\alpha=1}^{2}\left\|\left|a_{\alpha}\right|\right\|_{l+1 / 2, \gamma, \mathbb{R}_{\infty}^{2}}+\left|a_{3}\right|_{l, \gamma, \mathbb{R}_{\infty}^{2}}+\sigma\||A|\|_{l-1 / 2, \gamma, \mathbb{R}_{\infty}^{2}}\right. \\
& +\left\|\left|\boldsymbol{u}_{1}^{+}\right|\right\|_{2+l, \gamma, D_{\infty}^{+}}+\left\|\left|\boldsymbol{u}_{1}^{-}\right|\right\|_{2+l, \gamma, D_{\infty}^{-}}+\left\|\left|\boldsymbol{u}_{2}^{-}\right|\right\|_{2+l, \gamma, D_{\infty}^{-}} \\
& \left.+\left\|\left|\boldsymbol{w}^{\prime}\right|\right\|_{2+l, \gamma, D_{\infty}^{3}}+\||g|\|_{1+l, \gamma, D_{\infty}^{+}}\right\} .
\end{aligned}
$$


Taking the equivalence of norms $\||f|\|_{m, \gamma}$ and $\|f\|_{H_{\gamma}^{m, m / 2}}$ and also inequalities (3.9)-(3.12) into account, we arrive at the estimate (3.2). The existence of a solution of (3.1) is proved. Its uniqueness in the spaces $H_{\gamma}^{m, m / 2}, \gamma \geqslant \gamma_{0}>0$, follows from the triviality of the solution of problem (2.1) with homogeneous boundary conditions.

REMARK 3.2 We note that the symmetry transformation with respect to the plane $\left\{x_{3}=0\right\}$ makes the liquids change places. It is clear that this change preserves Theorems 2.4 and 3.1. Thus, our results on the linear problem are independent of the location of the liquids.

\section{Problems (1.8) and (1.10)}

Now we suppose that $\Omega^{+} \equiv \Omega_{0}^{+}$is contained in the ball $B_{d-2}, d<\infty$. We introduce the notation: $B_{d}^{ \pm} \equiv B_{d} \cap \Omega^{ \pm}, B_{d T}^{ \pm}=B_{d}^{ \pm} \times(0, T) ; \Omega^{-} \equiv \Omega_{0}^{-}$.

First, we consider system (1.10) with $\boldsymbol{u}=0$. We rewrite this problem with respect to the functions $v$ and $p$ :

$$
\begin{gathered}
\mathcal{D}_{t} \boldsymbol{v}-\left(\rho_{0}^{+}(x)\right)^{-1} \nabla \mathbb{T}^{\prime}(\boldsymbol{v})=\boldsymbol{f} \text { in } Q_{T}^{+}, \\
\mathcal{D}_{t} \boldsymbol{v}-v^{-} \nabla^{2} \boldsymbol{v}+\frac{1}{\rho_{0}^{-}} \nabla p=\boldsymbol{f}, \quad \nabla \cdot \boldsymbol{v}=r \quad \text { in } Q_{T}^{-}, \\
\left.\boldsymbol{v}\right|_{t=0}=\boldsymbol{w}_{0} \quad \text { in } \quad \Omega^{-} \cup \Omega^{+}, \quad \boldsymbol{v} \underset{|x| \rightarrow \infty}{\longrightarrow} 0, \quad p \underset{|x| \rightarrow \infty}{\longrightarrow} 0, \\
{\left.[\boldsymbol{v}]\right|_{G_{T}}=0,\left.\quad\left[\mu^{ \pm} \Pi_{0} \mathbb{S}(\boldsymbol{v}) \boldsymbol{n}_{0}\right]\right|_{G_{T}}=\Pi_{0} a,} \\
-\left.\left(p+\lambda^{+} \nabla \cdot \boldsymbol{v}\right)\right|_{\Gamma}+\left.\left[\mu^{ \pm} \boldsymbol{n}_{0} \cdot \mathbb{S}(\boldsymbol{v}) \boldsymbol{n}_{0}\right]\right|_{\Gamma}-\left.\sigma \boldsymbol{n}_{0} \cdot \Delta_{\Gamma} \int_{0}^{t} \boldsymbol{v}\right|_{\Gamma} \mathrm{d} \tau \\
=b+\sigma \int_{0}^{t} B \mathrm{~d} \tau, \quad t \in(0, T) .
\end{gathered}
$$

Here $\Delta_{\Gamma}$ is the Beltrami-Laplace operator on the given interface $\Gamma$ and $\boldsymbol{n}_{0}$ is its outward normal with respect to $\Omega_{0}^{+}$.

We formulate the existence theorem for (4.1) with homogeneous initial data.

THEOREM 4.1 Assume that $\boldsymbol{w}_{0}=0$ in (4.1). Moreover, let $\Gamma \in W_{2}^{3 / 2+l}, \rho_{0}^{+} \in W_{2}^{1+l}\left(\Omega^{+}\right)$for some $l>1 / 2,0<R_{0} \leqslant \rho_{0}^{+}(x) \leqslant R_{\infty}<\infty, x \in \Omega^{+}$, and let inequalities (1.9) hold. Then for arbitrary $\boldsymbol{f} \in \bigcup_{i=-,+} \boldsymbol{H}_{\gamma}^{l, l / 2}\left(Q_{T}^{i}\right), r \in H_{\gamma}^{1+l, 1 / 2+l / 2}\left(Q_{T}^{-}\right), r=\nabla \cdot \boldsymbol{R}, \boldsymbol{R} \in \boldsymbol{H}_{\gamma}^{0, l+1 / 2}\left(Q_{T}^{-}\right)$, $\boldsymbol{a} \in \boldsymbol{H}_{\gamma}^{l+1 / 2, l / 2+1 / 4}\left(G_{T}\right), \quad b \in H_{\gamma}^{l+1 / 2,1 / 2, l / 2}\left(G_{T}\right)$, and $A \in H_{\gamma}^{l-1 / 2, l / 2-1 / 4}\left(G_{T}\right)$ with $T \leqslant \infty$, problem (4.1) is uniquely solvable on the interval $(0, T]$ provided that $\gamma$ is large enough. Its solution $(\boldsymbol{v}, p)$ has the properties: $\boldsymbol{v} \in \bigcup_{i=-,+} \boldsymbol{H}_{\gamma}^{2+l, 1+l / 2}\left(Q_{T}^{i}\right), p \in H_{\gamma}^{l, l / 2}\left(B_{T}^{-}\right), \nabla p \in \boldsymbol{H}_{\gamma}^{l, l / 2}\left(Q_{T}^{-}\right)$, and satisfies the inequality

$$
\begin{aligned}
& \|\boldsymbol{v}\|_{\cup_{i} \boldsymbol{H}_{\gamma}^{2+l, 1+l / 2}\left(Q_{T}^{i}\right)}+\|\nabla p\|_{\boldsymbol{H}_{\gamma}^{l, l / 2}\left(Q_{T}^{-}\right)}+\|p\|_{H_{\gamma}^{l, l / 2}\left(B_{T}^{-}\right)} \leqslant c 1\|\boldsymbol{f}\|_{\cup_{i} \boldsymbol{H}_{\gamma}^{l, l / 2}\left(Q_{T}^{i}\right)} \\
& +\|r\|_{H_{\gamma}^{1+l, 1 / 2+l / 2}\left(Q_{T}^{-}\right)}+\|\boldsymbol{R}\|_{\boldsymbol{H}_{\gamma}^{0,1+l / 2}\left(Q_{T}^{-}\right)}+\|\boldsymbol{a}\|_{\boldsymbol{H}_{\gamma}^{l+1 / 2, l / 2+1 / 4}\left(G_{T}\right)} \\
& \left.+\|b\|_{H_{\gamma}^{l+1 / 2,1 / 2, l / 2}\left(G_{T}\right)}+\sigma\|B\|_{H_{\gamma}^{l-1 / 2, l / 2-1 / 4}\left(G_{T}\right)}\right\} \equiv c_{1} M
\end{aligned}
$$


where the constant $c_{1}$ is independent of $T$.

Proof. We prove this theorem in two steps. At first, we obtain the estimate (4.2) as an a priori one. Next, we show that system (4.1) with $\boldsymbol{w}_{0}=0$ has a unique solution belonging to the spaces $H_{\gamma}^{l, l / 2}$.

Step 1. We use the Schauder method to prove the validity of (4.2).

We first estimate the solution outside some ball containing $\Omega^{+}$. We multiply equations (4.1) by a smooth function $\eta: \eta=0$ in the ball $B_{d-1}$ and $\eta=1$ outside the ball $B_{d}$. Let us introduce the new unknown functions $\boldsymbol{u}=\boldsymbol{v} \eta$ and $q=p \eta$. It is necessary to evaluate a solution of the following Cauchy problem:

$$
\begin{aligned}
& \mathcal{D}_{t} \boldsymbol{u}-v^{-} \nabla^{2} \boldsymbol{u}+\frac{1}{\rho_{0}^{-}} \nabla q=\boldsymbol{f} \eta-v^{-}\left(\boldsymbol{v} \nabla^{2} \eta+2(\nabla \eta \cdot \nabla) \boldsymbol{v}\right)+\frac{1}{\rho_{0}^{-}} p \nabla \eta, \\
& \nabla \cdot \boldsymbol{v}=r \eta+\boldsymbol{v} \cdot \nabla \eta, \\
& \left.\boldsymbol{u}\right|_{t=0}=0, \quad \boldsymbol{u} \underset{|x| \rightarrow \infty}{\longrightarrow} 0, \quad q \underset{|x| \rightarrow \infty}{\longrightarrow} 0 .
\end{aligned}
$$

This problem was analysed in [2] where was obtained the estimate

$$
\begin{aligned}
& \|\boldsymbol{u}\|_{\boldsymbol{H}_{\gamma}^{2+l, 1+l / 2}\left(\mathbb{R}_{T}^{3}\right)}+\|\nabla q\|_{\boldsymbol{H}_{\gamma}^{l, l / 2}\left(\mathbb{R}_{T}^{3}\right)} \leqslant c_{2}\left\{\|\boldsymbol{f}\|_{\boldsymbol{H}_{\gamma}^{l, l / 2}\left(Q_{T}^{-}\right)}+\|r\|_{H_{\gamma}^{1+l, \frac{1+l}{2}}\left(Q_{T}^{-}\right)}+\|\boldsymbol{R}\|_{\boldsymbol{H}_{\gamma}^{0,1+l / 2}\left(Q_{T}^{-}\right)}\right. \\
& \left.+\|\boldsymbol{v}\|_{\boldsymbol{H}_{\gamma}^{2+l, 1+l / 2}\left(K_{d T}\right)}+\|p\|_{H_{\gamma}^{l, l / 2}\left(K_{d T}\right)}\right\}
\end{aligned}
$$

Here $K_{d T}=\left(B_{d} \backslash B_{d-1}\right) \times(0, T)$.

Next, by a partition of unity, on the base of local estimates for compressible [9] and incompressible [7] fluids, as well as of the boundary estimate (3.2) and inequality (4.3), we deduce that

$$
\begin{gathered}
\|\boldsymbol{v}\|_{\cup_{i} \boldsymbol{H}_{\gamma}^{2+l, 1+l / 2}\left(Q_{T}^{i}\right)}+\|\nabla p\|_{\boldsymbol{H}_{\gamma}^{l, l / 2}\left(Q_{T}^{-}\right)}+\|p\|_{H_{\gamma}^{l, l / 2}\left(B_{d T}^{-}\right)} \\
\leqslant c_{3}\left\{M+\|\boldsymbol{v}\|_{\bigcup_{i} \boldsymbol{H}_{\gamma}^{1+l, 1 / 2+l / 2}\left(B_{d T}^{i}\right)}+\|p\|_{H_{\gamma}^{l, l / 2}\left(B_{d T}^{-}\right)}+\|p\|_{H_{\gamma}^{0, l / 2}\left(G_{T}\right)}\right\} \\
\leqslant c_{4}\left\{M+\|\boldsymbol{v}\|_{\cup_{i} \boldsymbol{H}_{\gamma}^{1+l, 1 / 2+l / 2}\left(B_{d T}^{i}\right)}+\varepsilon\|\nabla p\|_{\boldsymbol{H}_{\gamma}^{l, l / 2}\left(B_{d T}^{-}\right)}\right. \\
\left.+c_{5}(\varepsilon)\|p\|_{H_{\gamma}^{0, l / 2}\left(B_{d T}^{-}\right)}\right\} .
\end{gathered}
$$

In the last relation, we have used interpolation inequalities from [2: p. 21].

Thus, it remains to estimate only $\|p\|_{H_{\gamma}^{0, l / 2}\left(B_{d T}^{-}\right)}$. To this end, we consider the Dirichlet problem

$$
\begin{gathered}
\frac{\nabla^{2} p}{\rho_{0}^{-}}=\nabla \cdot\left(\boldsymbol{f}-\mathcal{D}_{t} \boldsymbol{v}+v^{-} \nabla^{2} \boldsymbol{v}\right)=\nabla \cdot\left(\boldsymbol{f}-\mathcal{D}_{t} \boldsymbol{R}+v^{-} \nabla r\right) \\
p \nabla \cdot \boldsymbol{F} \quad \text { in } \Omega^{-} \\
\left.p\right|_{\Gamma}=\left.\left[2 \mu^{ \pm}\left(\frac{\partial \boldsymbol{v}}{\partial \boldsymbol{n}_{0}}\right)_{\boldsymbol{n}_{0}}\right]\right|_{\Gamma}-\left.\lambda^{+} \nabla \cdot \boldsymbol{v}\right|_{\Gamma}-\left.\sigma \boldsymbol{n}_{0} \cdot \Delta_{\Gamma} \int_{0}^{t} \boldsymbol{v}\right|_{\Gamma} \mathrm{d} \tau \\
-b-\sigma \int_{0}^{t} B \mathrm{~d} \tau \equiv p_{0}, \quad p \underset{|x| \rightarrow \infty}{\longrightarrow} 0
\end{gathered}
$$


where $\boldsymbol{b}_{\boldsymbol{n}_{0}}=\boldsymbol{b} \cdot \boldsymbol{n}_{0}$

We extend the functions $\boldsymbol{f}, r, \boldsymbol{R}, \boldsymbol{v}, b, B$ with preservation of class into the region $t>T$ in the case when $T<\infty$. After the Laplace transform $\widehat{f}(x, s)=\int_{0}^{\infty} \mathrm{e}^{-s t} f(x, t) \mathrm{d} t$ in (4.5)we arrive at the problem

$$
\begin{aligned}
\frac{\nabla^{2} \widehat{p}}{\rho_{0}^{-}}= & \nabla \cdot\left(\widehat{\boldsymbol{f}}-s \widehat{\boldsymbol{R}}+v^{-} \nabla \widehat{r}\right) \equiv \nabla \cdot \widehat{\boldsymbol{F}} \quad \text { in } \quad \Omega^{-}, \\
\left.\widehat{p}\right|_{\Gamma}= & {\left.\left[2 \mu^{ \pm}\left(\frac{\partial \widehat{\boldsymbol{v}}}{\partial \boldsymbol{n}_{0}}\right)_{\boldsymbol{n}_{0}}\right]\right|_{\Gamma}-\left.\lambda^{+} \nabla \cdot \widehat{\boldsymbol{v}}\right|_{\Gamma}-\left.\frac{\sigma}{s}\left(\Delta_{\Gamma} \widehat{\boldsymbol{v}}\right)_{\boldsymbol{n}_{0}}\right|_{\Gamma} } \\
& -\widehat{b}-\frac{\sigma}{s} \widehat{\boldsymbol{B}} \equiv \widehat{p}_{0}, \quad \widehat{p} \underset{|x| \rightarrow \infty}{\longrightarrow} 0 .
\end{aligned}
$$

We shall need also the solution $\Phi$ of the Dirichlet problem with homogeneous boundary condition

$$
\frac{\nabla^{2} \Phi}{\rho_{0}^{-}}=\overline{\bar{p}} \zeta^{2} \quad \text { in } \quad \Omega^{-},\left.\quad \Phi\right|_{\Gamma}=0, \quad \Phi \underset{|x| \rightarrow \infty}{\longrightarrow} 0 .
$$

Here $\zeta$ is a smooth function with compact support equal to 1 in $B_{d}$ and to 0 outside $B_{d+1}$. The bar means complex conjugation.

We multiply the equation in (4.7) by $\Phi$ and integrate by parts twice. Then we have

$$
\int_{\Omega^{-}}|\widehat{p}|^{2} \zeta^{2} \mathrm{~d} x=-\int_{\Omega^{-}} \widehat{\boldsymbol{F}} \cdot \nabla \Phi \mathrm{d} x+\int_{\Gamma} \widehat{p}_{0} \frac{\partial \Phi}{\partial \boldsymbol{n}_{0}} \mathrm{~d} \Gamma
$$

whence it follows that

$$
\|\widehat{p} \zeta\|_{\Omega^{-}}^{2} \leqslant c_{6}\left(\|\widehat{\boldsymbol{F}}\|_{\Omega^{-}}^{2}+\varepsilon\|\nabla \Phi\|_{\Omega^{-}}^{2}+\left\|\widehat{p}_{0}\right\|_{\Gamma}^{2}+\varepsilon\left\|\frac{\partial \Phi}{\partial \boldsymbol{n}_{0}}\right\|_{\Gamma}^{2}\right) .
$$

Applying the known estimates for $\left.\nabla \Phi\right|_{\Gamma}$ and $\nabla \nabla \Phi$

$$
\|\nabla \Phi\|_{\Gamma}^{2} \leqslant c_{7}\left(\|\nabla \nabla \Phi\|_{B_{d}^{-}}^{2}+\|\nabla \Phi\|_{\Omega^{-}}^{2}\right), \quad\|\nabla \nabla \Phi\|_{B_{d}^{-}}^{2} \leqslant c_{8}\left(\|\widehat{p} \zeta\|_{\Omega^{-}}^{2}+\|\nabla \Phi\|_{\Omega^{-}}^{2}\right),
$$

we see that it is only necessary to estimate $\|\nabla \Phi\|_{\Omega^{-}}^{2}$. To this end, we multiply the equation of (4.7) by $\bar{\Phi}$ and integrate by parts over $\Omega^{-}$. We then obtain the equality $\int_{\Omega^{-}} \frac{|\nabla \Phi|^{2}}{\rho_{0}^{-}} \mathrm{d} x=-\int_{\Omega^{-}} \bar{p} \zeta^{2} \bar{\Phi} \mathrm{d} x$ from which it follows that

$$
\|\nabla \Phi\|_{\Omega^{-}}^{2} \leqslant \varepsilon_{1}\|\Phi \zeta\|_{\Omega^{-}}^{2}+c_{\varepsilon_{1}}\|\widehat{p} \zeta\|_{\Omega^{-}}^{2} .
$$

Next, by the embedding theorems we obtain: $\|\Phi \zeta\|_{\Omega^{-}}^{2} \leqslant c_{9}\|\Phi \zeta\|_{L^{6}\left(B_{d+1}^{-}\right)}^{2} \leqslant c_{9}\|\nabla \Phi\|_{\Omega^{-}}^{2}$. Finally, choosing sufficiently small $\varepsilon_{1}$ and $\varepsilon$, we deduce from (4.9)

$$
\|\nabla \Phi\|_{\Omega^{-}}^{2} \leqslant c_{10}\|\widehat{p} \zeta\|_{\Omega^{-}}^{2},
$$


and then from (4.8)

$$
\begin{gathered}
\|\widehat{p} \zeta\|_{\Omega^{-}}^{2} \leqslant c_{11}\left(\|\widehat{\boldsymbol{F}}\|_{\Omega^{-}}^{2}+\left\|\widehat{p}_{0}\right\|_{\Gamma}^{2}\right) \leqslant c_{12}\left\{\|\widehat{\boldsymbol{f}}\|_{\Omega^{-}}^{2}+\|\widehat{\boldsymbol{r}}\|_{\Omega^{-}}^{2}+|s|^{2}\|\widehat{\boldsymbol{R}}\|_{\Omega^{-}}^{2}\right. \\
\left.+\|\nabla \widehat{\boldsymbol{v}}\|_{\Gamma}^{2}+\frac{\sigma^{2}}{|s|^{2}}\left\|\left(\Delta_{\Gamma} \widehat{\boldsymbol{v}}\right)_{\boldsymbol{n}_{0}}+\widehat{B}\right\|_{\Gamma}^{2}+\|\widehat{b}\|_{\Gamma}^{2}\right\} .
\end{gathered}
$$

Let us multiply inequality (4.10) by $|s|^{l}$ and integrate over the $\operatorname{line} \operatorname{Im} s=\xi_{0}$. By the Parseval equality, we obtain the estimate

$$
\begin{aligned}
\|p\|_{H_{\gamma}^{0, l / 2}\left(B_{d \infty}^{-}\right)}^{2} \leqslant & c_{13}\left\{\|\boldsymbol{f}\|_{\boldsymbol{H}_{\gamma}^{0, l / 2}\left(Q_{\infty}^{-}\right)}^{2}+\|r\|_{H_{\gamma}^{l / 2}\left(Q_{\infty}^{-}\right)}^{2}+\|\boldsymbol{R}\|_{\boldsymbol{H}_{\gamma}^{0,1+l / 2}\left(Q_{\infty}^{-}\right)}^{2}\right. \\
& +\|b\|_{H_{\gamma}^{0, l / 2}\left(G_{\infty}\right)}+\sigma^{2}\left\|\left(\Delta_{\Gamma} \boldsymbol{v}\right)_{\boldsymbol{n}_{0}}+B\right\|_{H_{\gamma}^{0, l / 2-1}\left(G_{\infty}\right)} \\
& \left.+\|\nabla \boldsymbol{v}\|_{H_{\gamma}^{0, l / 2}\left(G_{\infty}\right)}\right\}
\end{aligned}
$$

For $l<2$ the term with $\sigma^{2}$ must be replaced by $\sigma^{2} \gamma^{l-2}\left\|\mathrm{e}^{-\gamma t}\left[\left(\Delta_{\Gamma} \boldsymbol{v}\right)_{n_{0}}+B\right]\right\|_{G_{\infty}}$.

By means of the embedding theorems for $H_{\gamma}^{m, m / 2}$ [7], in the same way as in [2], we obtain from (4.4), (4.11) that

$$
\begin{aligned}
\|\boldsymbol{v}\|_{\bigcup_{i} \boldsymbol{H}_{\gamma}^{2+l, 1+l / 2}\left(Q_{T}^{i}\right)}+\|\nabla p\|_{\boldsymbol{H}_{\gamma}^{l, l / 2}\left(Q_{T}^{-}\right)} & +\|p\|_{H_{\gamma}^{0, l / 2}\left(B_{d T}^{-}\right)}^{2} \\
& \leqslant c_{14}\left\{M+\|\boldsymbol{v}\|_{\bigcup_{i} \boldsymbol{H}_{\gamma}^{3 / 2+j, 3 / 4+j / 2}\left(B_{d T}^{i}\right)}\right\} .
\end{aligned}
$$

Here $j=\max (1, l)$ which is always less than $l+1 / 2$, since $l>1 / 2$, and hence we have the weak norm of $\boldsymbol{v}$ on the right. It can be estimated by $\theta\|\boldsymbol{v}\|_{\bigcup_{i} \boldsymbol{H}_{\gamma}^{2+l, 1+l / 2}\left(Q_{T}^{i}\right)}$ with a small coefficient $\theta$ for a sufficiently large value of the parameter $\gamma$ (see [7: Section 4]). Thus, we arrive at estimate (4.2).

Step 2. The proof of existence of a solution with the mentioned properties is proved by constructing a regularizer in the same manner as in the case of single liquid [7,9]. The uniqueness of the constructed solution follows from the triviality of the homogeneous problem solution which is due to inequality (4.2).

The passage from the weighted spaces $H_{\gamma}^{m, m / 2}\left(Q_{T}^{ \pm}\right), \gamma \geqslant 0$, to the ordinary Sobolev spaces $W_{2}^{m, m / 2}\left(Q_{T}^{ \pm}\right)$is easily made because they are equivalente for $\forall T<\infty$ [7: Section 6].

Problem (4.1) with $\boldsymbol{w}_{0} \neq 0$ can be reduced to the problem with homogeneous initial conditions considered above by constructing a vector $\boldsymbol{V} \in \bigcup_{i} \boldsymbol{W}_{2}^{2+l, 1+l / 2}\left(Q_{T}^{i}\right)$ such that $\boldsymbol{V}(x, 0)=$ $\boldsymbol{w}_{0},\left.[\boldsymbol{V}]\right|_{G_{T}}=0$ and

$$
\|\boldsymbol{V}\|_{\bigcup_{i} \boldsymbol{W}_{2}^{2+l, 1+l / 2}\left(Q_{T}^{i}\right)} \leqslant c_{15}\left\|\boldsymbol{w}_{0}\right\|_{\bigcup_{i} \boldsymbol{W}_{2}^{1+l}\left(\Omega^{i}\right)} \cdot
$$

In this way, we arrive at Theorem 1.2 with $\boldsymbol{u}=0$.

Theorem 4.2 Suppose that $\Gamma \in W_{2}^{3 / 2+l}, \rho_{0}^{+} \in W_{2}^{1+l}\left(\Omega_{0}^{+}\right)$for some $l \in(1 / 2,1), 0<$ $R_{0} \leqslant \rho_{0}^{+}(x) \leqslant R_{\infty}<\infty, x \in \Omega_{0}^{+}$, and that inequalities (1.9) hold. In addition, assume that $\boldsymbol{f} \in \bigcup_{i=-,+} \boldsymbol{W}_{2}^{l, l / 2}\left(Q_{T}^{i}\right), r \in W_{2}^{1+l, 1 / 2+l / 2}\left(Q_{T}^{-}\right), r=\nabla \cdot \boldsymbol{R}, \boldsymbol{R} \in \boldsymbol{W}_{2}^{0,1+l / 2}\left(Q_{T}^{-}\right)$, 
$\boldsymbol{w}_{0} \in \bigcup_{i=-,+} \boldsymbol{W}_{2}^{1+l}\left(\Omega_{0}^{i}\right), \boldsymbol{a} \in \boldsymbol{W}_{2}^{l+1 / 2, l / 2+1 / 4}\left(G_{T}\right), b \in W_{2}^{l+1 / 2, l / 2+1 / 4}\left(G_{T}\right)$, and $B \in$ $W_{2}^{l-1 / 2, l / 2-1 / 4}\left(G_{T}\right)$ with $T<\infty$ and that the compatibility conditions

$$
\begin{gathered}
{\left.\left[\boldsymbol{w}_{0}\right]\right|_{\Gamma}=0,\left.\quad\left[\mu^{ \pm} \Pi_{0} \mathbb{S}\left(\boldsymbol{w}_{0}\right) \boldsymbol{n}_{0}\right]\right|_{\Gamma}=\left.\Pi_{0} \boldsymbol{a}\right|_{t=0},} \\
\nabla \cdot \boldsymbol{w}_{0}=\left.r\right|_{t=0} \text { on } \Omega_{0}^{-}
\end{gathered}
$$

are satisfied.

Then problem (4.1) is uniquely solvable and its solution $(\boldsymbol{v}, p)$ has the properties: $\boldsymbol{v} \in$ $\bigcup_{i=-,+} \boldsymbol{W}_{2}^{2+l, 1+l / 2}\left(Q_{T}^{i}\right), \quad p \in W_{2, l o c}^{l, l / 2}\left(Q_{T}^{-}\right), \nabla p \in \boldsymbol{W}_{2}^{l, l / 2}\left(Q_{T}^{-}\right),\left.p\right|_{G_{T}} \in W_{2}^{l+1 / 2, l / 2+1 / 4}\left(G_{T}\right)$, and

$$
\begin{aligned}
N[v, p] \equiv & \|\boldsymbol{v}\|_{Q_{T}^{-} \cup Q_{T}^{+}}^{(2+l, 1+l / 2)}+\|\nabla p\|_{Q_{T}^{-}}^{(l, l / 2)}+\|p\|_{B_{d T}^{-}}^{(l, l / 2)}+\|p\|_{W_{2}^{l+1 / 2, l / 2+1 / 4}\left(G_{T}\right)} \\
\leqslant c_{16}(T) & \left\{\boldsymbol{f}\left\|_{Q_{T}^{-} \cup Q_{T}^{+}}^{(l, l / 2)}+\right\| \boldsymbol{w}_{0}\left\|_{\cup_{i} \boldsymbol{W}_{2}^{1+l}\left(\Omega_{0}^{i}\right)}+\right\| r \|_{W_{2}^{1+l, 0}\left(Q_{T}^{-}\right)}\right. \\
& +\|\boldsymbol{R}\|_{W_{2}^{0,1+l / 2}\left(Q_{T}^{-}\right)}+T^{-l / 2}\left\|\mathcal{D}_{t} \boldsymbol{R}\right\|_{Q_{T}^{-}}+\|\boldsymbol{a}\|_{W_{2}^{l+1 / 2, l / 2+1 / 4}\left(G_{T}\right)} \\
& +\|b\|_{W_{2}^{l+1 / 2, l / 2+1 / 4}\left(G_{T}\right)}+T^{-l / 2}\|b\|_{W_{2}^{1 / 2,0}\left(G_{T}\right)} \\
& \left.+\sigma\|B\|_{W_{2}^{l-1 / 2, l / 2-1 / 4}\left(G_{T}\right)}\right\} \equiv c_{16}(T) F
\end{aligned}
$$

$c_{16}(T)$ being a non-decreasing function of $T$.

We will solve problem (1.10) by successive approximations taking $\boldsymbol{w}^{(0)}=0, s^{(0)}=0$ and determining $\left(\boldsymbol{w}^{(m+1)}, s^{(m+1)}\right), m \geqslant 0$, as solutions of the problems

$$
\begin{aligned}
& \mathcal{D}_{t} \boldsymbol{w}^{(m+1)}-\left(\rho_{0}^{+}(x)\right)^{-1} \nabla \mathbb{T}^{\prime}\left(\boldsymbol{w}^{(m+1)}\right)=\boldsymbol{f}+\boldsymbol{l}_{1}\left(\boldsymbol{w}^{(m)}\right) \text { in } Q_{T}^{+}, \\
& \mathcal{D}_{t} \boldsymbol{v}-v^{-} \nabla^{2} \boldsymbol{w}^{(m+1)}+\frac{1}{\rho_{0}^{-}} \nabla \boldsymbol{s}^{(m+1)}=\boldsymbol{f}+\boldsymbol{l}_{1}\left(\boldsymbol{w}^{(m)}, s^{(m)}\right), \\
& \nabla \cdot \boldsymbol{w}^{(m+1)}=r+l_{2}\left(\boldsymbol{w}^{(m)}\right)=\nabla \cdot\left(\mathcal{L}\left(\boldsymbol{w}^{(m)}\right)+\boldsymbol{R}\right) \quad \text { in } \quad Q_{T}^{-}, \\
& \left.\boldsymbol{w}^{(m+1)}\right|_{t=0}=\boldsymbol{w}_{0}, \quad \boldsymbol{w}^{(m+1)} \underset{|\xi| \rightarrow \infty}{\longrightarrow} 0, \quad s^{(m+1)} \underset{|\xi| \rightarrow \infty}{\longrightarrow} 0, \\
& {\left.\left[\boldsymbol{w}^{(m+1)}\right]\right|_{G_{T}}=0,\left.\quad\left[\mu^{ \pm} \Pi_{0} \mathbb{S}\left(\boldsymbol{w}^{(m+1)}\right) \boldsymbol{n}_{0}\right]\right|_{G_{T}}=\boldsymbol{l}_{3}\left(\boldsymbol{w}^{(m)}\right)+\Pi_{0} a,} \\
& {\left.\left[\boldsymbol{n}_{0} \cdot \mathbb{T}^{\prime}\left(\boldsymbol{w}^{(m+1)}, s^{(m+1)}\right) \boldsymbol{n}_{0}\right]\right|_{\Gamma}-\left.\sigma \boldsymbol{n}_{0} \cdot \Delta_{\Gamma} \int_{0}^{t} \boldsymbol{w}^{(m+1)}\right|_{\Gamma} \mathrm{d} \tau} \\
& =l_{4}\left(\boldsymbol{w}^{(m)}, s^{(m)}\right)+b+\sigma \int_{0}^{t}\left(l_{5}\left(\boldsymbol{w}^{(m)}\right)+B\right) \mathrm{d} \tau, \quad t \in(0, T) .
\end{aligned}
$$


Here we use the notation

$$
\begin{aligned}
& \boldsymbol{l}_{1}(\boldsymbol{w}, s)= \begin{cases}\left(\rho_{0}^{+}(\xi)\right)^{-1}\left[\mathbb{A} \nabla \mathbb{T}_{\boldsymbol{u}}^{\prime}(\boldsymbol{w})-\nabla \mathbb{T}^{\prime}(\boldsymbol{w})\right] & \text { in } \Omega_{t}^{+}, \\
v^{-}\left(\nabla_{\boldsymbol{u}}^{2}-\nabla^{2}\right) \boldsymbol{w}+\left(\nabla-\nabla_{\boldsymbol{u}}\right) s & \text { in } \Omega_{t}^{-},\end{cases} \\
& l_{2}(\boldsymbol{w})=\left(\nabla-\nabla_{\boldsymbol{u}}\right) \boldsymbol{w}=\nabla \cdot \mathcal{L}(\boldsymbol{w}), \quad \mathcal{L}(\boldsymbol{w})=\left(\mathbb{I}-\mathbb{A}^{T}\right) \boldsymbol{w}, \\
& \boldsymbol{l}_{3}(\boldsymbol{w})=\left.\left[\mu^{ \pm} \Pi_{0}\left(\mathbb{S}(\boldsymbol{w}) \boldsymbol{n}_{0}-\Pi_{\boldsymbol{u}} \mathbb{S}_{\boldsymbol{u}}(\boldsymbol{w}) \boldsymbol{n}\right)\right]\right|_{G_{T}}, \\
& l_{4}(\boldsymbol{w}, s)=\left.\left[\boldsymbol{n}_{0} \cdot\left(\mathbb{T}^{\prime}(\boldsymbol{w}, s) \boldsymbol{n}_{0}-\mathbb{T}_{\boldsymbol{u}}^{\prime}(\boldsymbol{w}, s) \boldsymbol{n}\right)\right]\right|_{G_{T}}, \\
& l_{5}(\boldsymbol{w})=\left.\boldsymbol{n}_{0} \cdot \mathcal{D}_{t}\left(\Delta(t)-\Delta_{\Gamma}\right) \int_{0}^{t} \boldsymbol{w}\right|_{\Gamma} \mathrm{d} \tau \\
& =\boldsymbol{n}_{0} \cdot\left\{\left(\Delta(t)-\Delta_{\Gamma}\right) \boldsymbol{w}+\left.\dot{\Delta}(t) \int_{0}^{t} \boldsymbol{w}\right|_{\Gamma} \mathrm{d} \tau\right\}
\end{aligned}
$$

where $\dot{\Delta}(t)$ is the derivative of $\Delta(t)$ with respect to time.

Operators $\boldsymbol{l}_{1}, \ldots, l_{5}$ and $\mathcal{L}$ were considered in [8] and [9] where the following estimates were obtained.

LEMMA 4.3 If $\boldsymbol{u}$ and $\boldsymbol{u}^{\prime}$ satisfy inequality (1.11) then

$$
\begin{aligned}
& \left\|\boldsymbol{l}_{1}(\boldsymbol{w}, s)-\boldsymbol{l}_{1}^{\prime}(\boldsymbol{w}, s)\right\|_{Q_{T}^{-} \cup Q_{T}^{+}}^{(l, l / 2)}+\left\|l_{2}(\boldsymbol{w})-l_{2}^{\prime}(\boldsymbol{w})\right\|_{W_{2}^{1+l, \frac{1+l}{2}}}^{(l, l / 2)}\left(Q_{T}^{-}\right) \\
& +\left\|\boldsymbol{l}_{3}(\boldsymbol{w})-\boldsymbol{l}_{3}^{\prime}(\boldsymbol{w})\right\|_{W_{2}^{1 / 2+l, 1 / 4+l / 2}\left(G_{T}\right)}+\left\|l_{5}(\boldsymbol{w})-l_{5}^{\prime}(\boldsymbol{w})\right\|_{G_{T}}^{(l-1 / 2, l / 2-1 / 4)}
\end{aligned}
$$

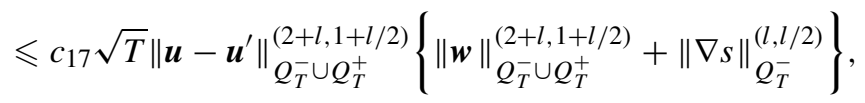

$$
\begin{aligned}
& \left\|\mathcal{D}_{t}\left(\mathcal{L}(\boldsymbol{w})-\mathcal{L}^{\prime}(\boldsymbol{w})\right)\right\|_{Q_{T}^{-}}^{(0, l / 2)} \leqslant c_{18}\left\{\sqrt{T}\left\|\boldsymbol{u}-\boldsymbol{u}^{\prime}\right\|_{Q_{T}^{-}}^{(2+l, 1+l / 2)}\right. \\
& \left.+T^{\frac{1-l}{2}}\left\|\boldsymbol{u}(\cdot, 0)-\boldsymbol{u}^{\prime}(\cdot, 0)\right\|_{\boldsymbol{W}_{2}^{1}\left(\Omega^{-}\right)}\right\}\|\boldsymbol{w}\|_{Q_{T}^{-}}^{(2+l, 1+l / 2)}, \\
& \left\|l_{4}(\boldsymbol{w}, s)-l_{4}^{\prime}(\boldsymbol{w}, s)\right\|_{W^{1 / 2+l, 1 / 4+l / 2}\left(G_{T}\right)} \leqslant c_{19} \sqrt{T}\left\|\boldsymbol{u}-\boldsymbol{u}^{\prime}\right\|_{Q_{T}^{-} \cup Q_{T}^{+}}^{(2+l, 1+l / 2)} \\
& \times\left\{\|\boldsymbol{w}\|_{Q_{T}^{-} \cup Q_{T}^{+}}^{(2+l, 1+l / 2)}+\|\nabla s\|_{Q_{T}^{-}}^{(l, l / 2)}+\|s\|_{B_{T}^{-}}^{(l, l / 2)}+\|s\|_{W_{2}^{l+1 / 2, l / 2+1 / 4}\left(G_{T}\right)}\right\} .
\end{aligned}
$$

Here operators $\boldsymbol{l}_{1}^{\prime}, \ldots, l_{5}^{\prime}$ and $\mathcal{L}^{\prime}$ are calculated according to formulas (4.14) where vector $\boldsymbol{u}$ is replaced by $\boldsymbol{u}^{\prime}$. If $\left.\boldsymbol{w}\right|_{t=0}=0$, then inequality (4.15) is valid without $T^{\frac{1-l}{2}}\left\|\boldsymbol{u}(\cdot, 0)-\boldsymbol{u}^{\prime}(\cdot, 0)\right\|_{\boldsymbol{W}_{2}^{1}\left(\Omega^{-}\right)}$ in the right-hand side.

REMARK 4.4 We note also that in (4.16) it is sufficient to take the norm $\|s\|^{(l, l / 2)}$ over the bounded domain $B_{T}^{-}$.

Lemma 4.5 follows on from Lemma 4.3. 
LEMMA 4.5 If $\boldsymbol{u}$ satisfies inequality (1.11), then

$$
\begin{aligned}
& \left\|\boldsymbol{l}_{1}(\boldsymbol{w}, s)\right\|_{Q_{T}^{-} \cup Q_{T}^{+}}^{(l, l / 2)}+\left\|l_{2}(\boldsymbol{w})\right\|_{W_{2}^{1+l, \frac{1+l}{2}}\left(Q_{T}^{-}\right)}^{(l, l / 2)}+\left\|\boldsymbol{l}_{3}(\boldsymbol{w})\right\|_{W_{2}^{1 / 2+l, 1 / 4+l / 2}\left(G_{T}\right)} \\
& +\left\|l_{4}(\boldsymbol{w}, s)\right\|_{W^{1 / 2+l, 1 / 4+l / 2}\left(G_{T}\right)}+\left\|l_{5}(\boldsymbol{w})\right\|_{G_{T}}^{(l-1 / 2, l / 2-1 / 4)} \\
& \leqslant c_{20} \delta\left\{\|\boldsymbol{w}\|_{Q_{T}^{-} \cup Q_{T}^{+}}^{(2+l, 1+l / 2)}+\|\nabla s\|_{Q_{T}^{-}}^{(l, l / 2)}+\|s\|_{B_{T}^{-}}^{(l, l / 2)}+\|s\|_{W_{2}^{l+1 / 2, l / 2+1 / 4}\left(G_{T}\right)}\right\}, \\
& \left\|\mathcal{D}_{t} \mathcal{L}(\boldsymbol{w})\right\|_{Q_{T}^{-}}^{(0, l / 2)} \leqslant c_{21}\left\{\delta+T^{\frac{1-l}{2}}\|\boldsymbol{u}(\cdot, 0)\|_{W_{2}^{1}\left(\Omega^{-}\right)}\right\}\|\boldsymbol{w}\|_{Q_{T}^{-}}^{(2+l, 1+l / 2)} .
\end{aligned}
$$

If $\boldsymbol{w}(\cdot, 0)=0$ in $\Omega^{-}$, then the term with $\|\boldsymbol{u}(\cdot, 0)\|_{\boldsymbol{W}_{2}^{1}\left(\Omega^{-}\right)}$may be dropped in the last inequality.

Proof of Theorem 1.2. We return to problem (4.13). It follows from Theorem 4.2 and Lemma 4.5 that $\left(\boldsymbol{w}^{(m+1)}, s^{(m+1)}\right), m>0$, are uniquely determined, $\left(\boldsymbol{w}^{(1)}, s^{(1)}\right)$ being a solution of (1.10) with $\boldsymbol{u}=0$ and satisfying inequality (4.12).

Let us consider the differences $\boldsymbol{z}^{(m+1)}=\boldsymbol{w}^{(m+1)}-\boldsymbol{w}^{(m)}, g^{(m+1)}=s^{(m+1)}-s^{(m)}, m \geqslant 1$. We have for them the problem

$$
\begin{aligned}
& \mathcal{D}_{t} \boldsymbol{z}^{(m+1)}-\left(\rho_{0}^{+}(x)\right)^{-1} \nabla \mathbb{T}^{\prime}\left(\boldsymbol{z}^{(m+1)}\right)=\boldsymbol{l}_{1}\left(\boldsymbol{z}^{(m)}\right) \quad \text { in } \quad Q_{T}^{+}, \\
& \mathcal{D}_{t} v-v^{-} \nabla^{2} \boldsymbol{z}^{(m+1)}+\frac{1}{\rho_{0}^{-}} \nabla g^{(m+1)}=\boldsymbol{l}_{1}\left(\boldsymbol{z}^{(m)}, g^{(m)}\right), \\
& \nabla \cdot \boldsymbol{z}^{(m+1)}=l_{2}\left(\boldsymbol{z}^{(m)}\right)=\nabla \cdot \mathcal{L}\left(\boldsymbol{z}^{(m)}\right) \text { in } Q_{T}^{-}, \\
& \left.\boldsymbol{z}^{(m+1)}\right|_{t=0}=0, \quad \boldsymbol{z}^{(m+1)} \underset{|\xi| \rightarrow \infty}{\longrightarrow} 0, \quad g^{(m+1)} \underset{|\xi| \rightarrow \infty}{\longrightarrow} 0, \\
& {\left.\left[\boldsymbol{z}^{(m+1)}\right]\right|_{G_{T}}=0,\left.\quad\left[\mu^{ \pm} \Pi_{0} \mathbb{S}\left(\boldsymbol{z}^{(m+1)}\right) \boldsymbol{n}_{0}\right]\right|_{G_{T}}=\boldsymbol{l}_{3}\left(\boldsymbol{z}^{(m)}\right)} \\
& {\left.\left[\boldsymbol{n}_{0} \cdot \mathbb{T}^{\prime}\left(\boldsymbol{z}^{(m+1)}, g^{(m+1)}\right) \boldsymbol{n}_{0}\right]\right|_{\Gamma}-\left.\sigma \boldsymbol{n}_{0} \cdot \Delta_{\Gamma} \int_{0}^{t} \boldsymbol{z}^{(m+1)}\right|_{\Gamma} \mathrm{d} \tau} \\
& =l_{4}\left(\boldsymbol{z}^{(m)}, g^{(m)}\right)+\sigma \int_{0}^{t} l_{5}\left(\boldsymbol{z}^{(m)}\right) \mathrm{d} \tau, \quad t \in(0, T) .
\end{aligned}
$$

If $m \geqslant 1$, then $\left.z^{(m+1)}\right|_{t=0}=0$, and we deduce from (4.12) and Lemma 4.5 that

$$
N\left[z^{(m+1)}, g^{(m+1)}\right] \leqslant c_{22} \delta N\left[z^{(m)}, g^{(m)}\right] .
$$

If $m=1$ then in virtue of (4.17) we obtain

$$
N\left[z^{(2)}, g^{(2)}\right] \leqslant\left(c_{22} \delta+c_{21} \delta_{1}\right) N\left[\boldsymbol{w}^{(1)}, s^{(1)}\right]
$$

with $\delta_{1}=T^{\frac{1-l}{2}}\|\boldsymbol{u}(\cdot, 0)\|_{\boldsymbol{W}_{2}^{1}\left(\Omega^{-}\right)}$. Next, for $\Sigma_{m}=\sum_{j=2}^{m} N\left[z^{(j)}, g^{(j)}\right]$ the following inequality

$$
\Sigma_{m+1} \leqslant c_{22} \delta \Sigma_{m}+N\left[z^{(2)}, g^{(2)}\right]
$$


holds. Let us take $c_{22} \delta<1$. It is obvious that

$$
\Sigma_{m+1} \leqslant\left(1-c_{22} \delta\right)^{-1} N\left[z^{(2)}, g^{(2)}\right] .
$$

Because of Theorem 4.2 we have:

$$
\begin{aligned}
N\left[\boldsymbol{w}^{(m+1)}, s^{(m+1)}\right] & \leqslant \Sigma_{m+1}+N\left[\boldsymbol{w}^{(1)}, s^{(1)}\right] \\
& \leqslant\left(\frac{1}{1-c_{22} \delta}+\frac{c_{21}}{1-c_{22} \delta} T^{\frac{1-l}{2}}\|\boldsymbol{u}(\cdot, 0)\|_{W_{2}^{1}\left(\Omega^{-}\right)}\right) c_{16} F
\end{aligned}
$$

where $F$ is the sum of the right-hand-side norms in (4.12). Hence, the sequence $\left\{\boldsymbol{w}^{(m+1)}, s^{(m+1)}\right\}$ is convergent and its limit $(\boldsymbol{w}, s)$ is a solution of (1.10) satisfying inequality (1.12) with

$$
c_{1}(T)=\frac{c_{16} F}{1-c_{22} \delta}\left(1+c_{21} T^{\frac{1-l}{2}}\|\boldsymbol{u}(\cdot, 0)\|_{W_{2}^{1}\left(\Omega^{-}\right)}\right) .
$$

In a similar way, we can conclude that the difference $\left(z=w-\boldsymbol{w}^{\prime}, g=s-s^{\prime}\right)$ of two solutions of (1.10) satisfies the estimate

$$
N[z, g] \leqslant c_{22} \delta N[z, g]
$$

whence it follows $z=0, g=0$. Thus, the uniqueness of the solution is also proved.

Now, we give the main ideas of the Proof of Theorem 1.1.

Using the formula for twice the mean curvature,

$$
\Delta(t) \boldsymbol{X}_{\boldsymbol{u}}=\Delta(0) \boldsymbol{\xi}+\int_{0}^{t} \dot{\Delta}(\tau) \boldsymbol{\xi} \mathrm{d} \tau+\Delta(t) \int_{0}^{t} \boldsymbol{u} \mathrm{d} \tau
$$

we rewrite the last boundary condition in (1.8) as follows:

$$
\begin{aligned}
{\left.\left[\boldsymbol{n}_{0} \cdot \mathbb{T}_{\boldsymbol{u}}^{\prime}(\boldsymbol{u}, q) \boldsymbol{n}\right]\right|_{G_{T}}-\left.\sigma \boldsymbol{n}_{0} \cdot \Delta(t) \int_{0}^{t} \boldsymbol{u}\right|_{G_{T}}=} & \sigma H_{0}(\xi)+\sigma \int_{0}^{t} \boldsymbol{n}_{0} \cdot \dot{\Delta}(\tau) \boldsymbol{\xi} \mathrm{d} \tau \\
& +\left.\left(\boldsymbol{n}_{0} \cdot \boldsymbol{n}\right) p^{+}\left(\rho_{0}^{+} \mathcal{J}_{\boldsymbol{u}}^{-1}\right)\right|_{G_{T}}
\end{aligned}
$$

Au.Q. open face ' $\mathbb{N}$ ? $\quad$ Here $H_{0}(\xi)=\boldsymbol{n}_{0} \cdot \Delta(0) \boldsymbol{\xi}$ is twice the mean curvature of $\Gamma$.

Next, we use again the successive approximation method, now for solving system (1.8). We put 
$\boldsymbol{u}^{(0)}=0, q^{(0)}=0$ and define $\boldsymbol{u}^{(m+1)}, q^{(m+1)}, m \in \mathbb{N}$, as a solution of the problem

$$
\begin{gathered}
\mathcal{D}_{t} \boldsymbol{u}^{(m+1)}-\frac{1}{\rho_{0}^{+}(\xi)} \mathbb{A}_{m} \nabla \mathbb{T}_{m}^{\prime}\left(\boldsymbol{u}^{(m+1)}\right)=\boldsymbol{f}\left(X_{m}, t\right)-\frac{1}{\rho_{0}^{+}(\xi)} \mathbb{A}_{m} \nabla p^{+}\left(\rho_{0}^{+} \mathcal{J}_{m}^{-1}\right) \text { in } \quad Q_{T}^{+}, \\
\mathcal{D}_{t} \boldsymbol{u}^{(m+1)}-v^{-} \nabla_{m}^{2} \boldsymbol{u}^{(m+1)}+\frac{1}{\rho^{-}} \nabla_{m} q^{(m+1)}=\boldsymbol{f}\left(X_{m}, t\right), \nabla_{m} \cdot \boldsymbol{u}^{(m+1)}=0 \quad \text { in } \quad Q_{T}^{-}, \quad \\
\left.\boldsymbol{u}^{(m+1)}\right|_{t=0}=\boldsymbol{v}_{0} \quad \text { in } \quad \Omega_{0}^{-} \cup \Omega_{0}^{+}, \quad \boldsymbol{u}^{(m+1)} \underset{|\xi| \rightarrow \infty}{\longrightarrow} 0, \quad q^{(m+1)} \underset{|\xi| \rightarrow \infty}{\longrightarrow} 0 \\
{\left.\left[\boldsymbol{u}^{(m+1)}\right]\right|_{G_{T}}=0,\left.\quad\left[\mu^{ \pm} \Pi_{0} \Pi_{m} \mathbb{S}_{m}\left(\boldsymbol{u}^{(m+1)}\right) \boldsymbol{n}_{m}\right]\right|_{G_{T}}=0} \\
{\left.\left[\boldsymbol{n}_{0} \cdot \mathbb{T}_{m}^{\prime}\left(\boldsymbol{u}^{(m+1)}, q^{(m+1)}\right) \boldsymbol{n}_{m}\right]\right|_{G_{T}}-\left.\sigma \boldsymbol{n}_{0} \cdot \Delta_{m}(t) \int_{0}^{t} \boldsymbol{u}^{(m+1)}\right|_{G_{T}}} \\
=\sigma H_{0}(\xi)+\sigma \int_{0}^{t} \boldsymbol{n}_{0} \cdot \dot{\Delta}_{m}(\tau) \boldsymbol{\xi} \mathrm{d} \tau+\left.\left(\boldsymbol{n}_{0} \cdot \boldsymbol{n}_{m}\right) p^{+}\left(\rho_{0}^{+} \mathcal{J}_{m}^{-1}\right)\right|_{G_{T}}
\end{gathered}
$$

Here we have used the notation $\nabla_{m}=\nabla_{\boldsymbol{u}^{(m)}}$ etc.; $\Delta_{m}(t)$ is the Beltrami-Laplace operator on $\Gamma_{m}(t)$; $\boldsymbol{n}_{m}$ is an outward normal to the surface $\Gamma_{m}(t)=\left\{x=X_{m}(\xi, t) \mid \xi \in \Gamma\right\} ; \Pi_{m}$ is the projector on its tangential plane; $\mathbb{A}_{m}$ is the cofactor matrix for the Jacobi matrix $\left\{a_{i j}^{(m)}\right\}$ of (1.5) corresponding to the vector field $\boldsymbol{u}^{(m)}$.

Since $\boldsymbol{n}_{0} \cdot \dot{\Delta}_{m}(\tau) \boldsymbol{\xi} \in W_{2}^{l-1 / 2, l / 2-1 / 4}\left(G_{T}\right)$ if $\boldsymbol{u}^{(m)} \in \cup_{i} \boldsymbol{W}_{2}^{2+l, 1+l}\left(Q_{T}^{i}\right)$ [8], and since $H_{0} \in$ $W_{2}^{l+1 / 2}(\Gamma)$ then, by Theorem 1.2, problem (4.19) is solvable in an interval $\left(0, T_{m}\right)$ in which $\boldsymbol{u}^{(m)}, q^{(m)}$ are determined and $\boldsymbol{u}^{(m)}$ satisfies condition (1.11) with a sufficiently small $\delta>0$. It is necessary to show that for $\forall m T_{m} \geqslant T^{\prime}>0, N\left[\boldsymbol{u}^{(m)}, q^{(m)}\right]$ are uniformly bounded in $\left(0, T^{\prime}\right)$ and that the sequence $\left\{\boldsymbol{u}^{(m)}, q^{(m)}\right\}$ converges to a solution of problem (1.8). The proof of these facts is the same as in the case of a single fluid. It was presented in detail in [8].

\section{Acknowledgments}

The author is grateful to the European Science Foundation Program 'Mathematical Treatment of Free Boundary Problems' for the financial support.

\section{REFERENCES}

1. Agranovich, M. S. \& Vishik, M. I. Elliptic problems with a parameter and parabolic problems of general type. Usp. Mat. Nauk 19(3), (1964) 53-152. (in Russian).

2. Denisova, I. V. A priori estimates of the solution of a linear time-dependent problem connected with the motion of a drop in a fluid medium. Trudy Mat. Inst. Steklov 188, (1990) 3-21. (English translation in Proc. Steklov Intit. Math. 3, (1991) 1-24.)

3. Denisova, I. V. Problem of the motion of two viscous incompressible fluids separated by a closed free interface. Acta Appl. Math. 37, (1994) 31-40.

4. Denisova, I. V. Problem of the motion of two compressible fluids separated by a closed free interface. Zap. Nauchn. Semin. Peterburg. Otdel. Mat. Inst. Steklov. (POMI) 243, (1997) 61-86. (English translation in J. Math. Sci. 99 (1), (2000) 837-853.)

5. Denisova, I. V. \& Solonnikov, V. A. Solvability of the linearized problem on the motion of a drop in a liquid flow. Zap. Nauchn. Semin. Leningrad. Otdel. Mat. Inst. Steklov. (LOMI) 171, (1989) 53-65. (English translation in J. Soviet Math. 56(2), (1991) 2309-2316.) 
6. Schlichting, H. Boundary Layer Theory. McGraw Hill, New York (1960).

7. SolonniKov, V. A. On an initial-boundary value problem for the Stokes systems arising in the study of a problem with a free boundary. Trudy Mat. Inst. Steklov. 188, (1990) 150-188. (English translation in Proc. Steklov Inst. Math. 3, (1991) 191-239.)

8. Solonnikov, V. A. Solvability of the problem of evolution of a viscous incompressible fluid bounded by a free surface on a finite time interval. Algebra i Analiz 3(1), (1991) 222-257. (English translation in St.Petersburg Math. J. 3(1), (1992) 189-220.)

9. Solonnikov, V. A. \& TANi, A. Free boundary problem for a viscous compressible flow with surface tension. Constantin Carathéodory: An International Tribute. pp. 1270-1303. World Scientific, (1991). 